\title{
MAXIMAL RANK OF SPACE CURVES IN THE RANGE A
}

\author{
EDOARDO BALLICO, PHILIPPE ELLIA, CLAUDIO FONTANARI
}

\begin{abstract}
We prove the following statement, which has been conjectured since 1985: There exists a constant $K$ such that for all natural numbers $d, g$ with $g \leq K d^{3 / 2}$ there exists an irreducible component of the Hilbert scheme of $\mathbb{P}^{3}$ whose general element is a smooth, connected curve of degree $d$ and genus $g$ of maximal rank.
\end{abstract}

\section{INTRODUCTION}

The postulation of algebraic space curves has been the object of wide interest in the last thirty years (see for instance [1], 2], 28, 22, 27]). In particular, the following Conjecture was stated in 1985 in [2], p. 2 (see also [3], §6, Problem 4):

Conjecture 1. There exists a constant $K$ such that for all natural numbers $d, g$ with $g \leq K d^{3 / 2}$ there exists an irreducible component of the Hilbert scheme of $\mathbb{P}^{3}$ whose general element is a smooth, connected curve of degree $d$ and genus $g$ of maximal rank.

We recall that a space curve $C$ is of maximal rank if the natural maps $H^{0}\left(\mathcal{O}_{\mathbb{P}^{3}}(m)\right) \rightarrow$ $H^{0}\left(\mathcal{O}_{C}(m)\right)$ are either injective or surjective for every $m$.

Here we consider smooth and connected curves $X$ with $h^{1}\left(\mathcal{I}_{X}(m)\right)=0, h^{0}\left(\mathcal{I}_{X}(m-\right.$ $1))=0, \operatorname{deg}(X)=d, g(X)=g$ and $h^{1}\left(\mathcal{O}_{X}(m-2)\right)=0$ (hence of maximal rank by Castelnuovo-Mumford regularity). Since $h^{1}\left(\mathcal{I}_{X}(m)\right)=0$ and $h^{1}\left(\mathcal{O}_{X}(m)\right)=0$, we have

$$
1+m d-g \leq\left(\begin{array}{c}
m+3 \\
3
\end{array}\right)
$$

Let $d(m, g)_{\max }$ be the maximal integer $d$ such that (10) is satisfied, i.e. set $\left.d(m, g)_{\max }:=\left\lfloor\left(\begin{array}{c}m+3 \\ 3\end{array}\right)+g-1\right) / m\right\rfloor$. Since $h^{0}\left(\mathcal{I}_{X}(m-1)\right)=0$ and $h^{1}\left(\mathcal{O}_{X}(m-1)\right)=0$, we have

$$
1+(m-1) d-g \geq\left(\begin{array}{c}
m+2 \\
3
\end{array}\right)
$$

Let $d(m, g)_{\min }$ be the minimal integer $d$ such that (2) is satisfied, i.e. set $\left.d(m, g)_{\min }:=\left\lceil\left(\begin{array}{c}m+2 \\ 3\end{array}\right)+g-1\right) /(m-1)\right\rceil$.

2010 Mathematics Subject Classification. Primary: 14H50. Secondary: 14N05.

Key words and phrases. Space curve. Postulation. Hilbert function. Hilbert scheme.

This research was partially supported by PRIN 2012 "Geometria delle varietà algebriche", by FIRB 2012 "Moduli spaces and Applications", and by GNSAGA of INdAM (Italy). 
For every integer $s>0$ define the number $p_{a}\left(C_{s}\right):=s(s+1)(2 s-5) / 6+1$ (which is going to to be the genus of the curve $C_{s}$ to be introduced later in Section 22). For all positive integers $m \geq 3$ set

$$
\begin{aligned}
\varphi(m)= & p_{a}\left(C_{\lfloor m / \sqrt{20}\rfloor-4}\right)+p_{a}\left(C_{\lfloor m / \sqrt{20}\rfloor-5}\right) \\
= & \frac{(\lfloor m / \sqrt{20}\rfloor-4)(\lfloor m / \sqrt{20}\rfloor-3)(2\lfloor m / \sqrt{20}\rfloor-13)}{6}+1 \\
& +\frac{(\lfloor m / \sqrt{20}\rfloor-5)(\lfloor m / \sqrt{20}\rfloor-4)(2\lfloor m / \sqrt{20}\rfloor-15)}{6}+1 .
\end{aligned}
$$

For any smooth curve $X \subset \mathbb{P}^{3}$ let $N_{X}$ denote the normal bundle of $X$ in $\mathbb{P}^{3}$. If $h^{1}\left(N_{X}\right)=0$, then $X$ is a smooth point of the Hilbert scheme of $\mathbb{P}^{3}$ and this Hilbert scheme has the expected dimension $h^{0}\left(N_{X}\right)$ at $X$.

Our main result is the following:

Theorem 1. For every integer $m \geq 3$ and every $(d, g)$ with $17052 \leq g \leq \varphi(m)$ and $d(m, g)_{\min } \leq d \leq d(m, g)_{\max }$ there exists a component of the Hilbert scheme of curves in $\mathbb{P}^{3}$ of genus $g$ and degree $d$, whose general element $X$ is smooth and satisfies $h^{0}\left(\mathcal{I}_{X}(m-1)\right)=0, h^{1}\left(\mathcal{I}_{X}(m)\right)=0, h^{1}\left(\mathcal{O}_{X}(m-2)\right)=0$, and $h^{1}\left(N_{X}(-1)\right)=0$.

As an application of Theorem 1 we prove Conjecture 1 Indeed, if $g=0$ we have just to quote [19]. Next, if $0<g<17052$ we may choose $K>0$ such that $g \geq K(g+3)^{3 / 2}$. Hence from $K(g+3)^{3 / 2} \leq g \leq K d^{3 / 2}$ we get $d \geq g+3$ and we are done by [1]. Finally, if $g \geq 17052$ we have the following:

Corollary 1. Let $K=\frac{2}{3}\left(\frac{1}{10}\right)^{3 / 2}$ and $\varepsilon=\frac{11}{20}+4\left(\frac{1}{20}\right)^{3 / 2}$. If $17052 \leq g \leq K d^{3 / 2}-$ $6 \varepsilon d$ then there exists an irreducible component of the Hilbert scheme of $\mathbb{P}^{3}$ whose general element $X$ is a smooth, connected curve of degree $d$ and genus $g$ of maximal rank and with $h^{1}\left(N_{X}(-1)\right)=0$.

The constant $K$ in Corollary 1 is certainly not optimal, but the exponent $d^{3 / 2}$ is sharp among the curves with $h^{1}\left(N_{X}\right)=0$ (see [12], 29, Corollaire 5.18] and [20, II.3.6] for the condition $h^{1}\left(N_{X}(-2)\right)=0$, 20, II.3.7] and 31] for the condition $h^{1}\left(N_{X}(-1)\right)=0$, and [20, II.3.8] for the condition $\left.h^{1}\left(N_{X}\right)=0\right)$.

If $X$ is as in Theorem 1, then by Castelnuovo-Mumford regularity we have $h^{1}\left(\mathcal{I}_{X}(t)\right)=0$ for all $t>m$ and the homogeneous ideal of $X$ is generated by forms of degree $m$ and degree $m+1$. A smooth curve $Y \subset \mathbb{P}^{3}$ with $h^{0}\left(\mathcal{I}_{Y}(m-1)\right)=$ $0, \frac{m^{2}+4 m+6}{6} \leq \operatorname{deg}(Y)<\frac{m^{2}+4 m+6}{3}$ and maximal genus among the curves with $h^{0}\left(\mathcal{I}_{Y}(m-1)\right)=0$ satisfies $h^{1}\left(\mathcal{O}_{Y}(m-1)\right)=0([16$, proof of Theorem 3.3 at p. 97]). In the statement of Theorem 1 we claim one shift more, namely, $h^{1}\left(\mathcal{O}_{X}(m-2)\right)=0$, in order to apply Castelnuovo-Mumford regularity to $X$.

We describe here one of the main differences with respect to [19, 1, 2]. Fix integers $d, g$ as in Theorem 1 or Corollary 1. Suppose that we have constructed two irreducible and generically smooth components $W_{1}, W_{2}$ of the Hilbert scheme of smooth space curves of degree $d$ and genus $g$. Suppose also that we have proved the existence of $Y_{1} \in W_{1}$ and $Y_{2} \in W_{2}$ with $h^{0}\left(\mathcal{I}_{Y_{2}}(m-1)\right)=0, h^{1}\left(\mathcal{I}_{Y_{1}}(m)\right)=0$ and $h^{1}\left(N_{Y_{i}}\right)=h^{1}\left(\mathcal{O}_{Y_{i}}(m-3)\right)=0, i=1,2$. If $W_{1}=W_{2}$, then by the semicontinuity theorem for cohomology and Castelnuovo-Mumford regularity a general $X \in W_{1}$ satisfies $h^{0}\left(\mathcal{I}_{X}(m-1)\right)=0, h^{1}\left(\mathcal{I}_{X}(t)\right)=0$ for all $t \geq m$ and $h^{1}\left(N_{X}\right)=0$. In particular a general element of $W_{1}$ has maximal rank. But we need to know that $W_{1}=W_{2}$. If $d \geq g+3$ it was not known at that time that the Hilbert scheme of 
smooth space curves of degree $d$ and genus $g$ is irreducible ([7]), but it was obvious since at least Castelnuovo that its part parametrizing the non-special curves is irreducible (modulo the irreducibility of the moduli scheme $\mathcal{M}_{g}$ of genus $g$ smooth curves). When $d<g+3$, the Hilbert scheme of smooth space curves of degree $d$ and genus $g$ is often reducible, even in ranges with $d / g$ not small $([6,21,23,24,25,26])$. In 2] when $d \geq(g+2) / 2$ we defined a certain irreducible component $Z(d, g)$ of the Hilbert scheme of smooth space curves of degree $d$ and genus $g$ and (under far stronger assumptions on $d, g$ ) we were able find $Y_{1}$ and $Y_{2}$ with $W_{1}=W_{2}=Z(d, g)$. Several pages of Section 5 are devoted to solve this problem.

We work over an algebraically closed field $\mathbb{K}$ of characteristic zero.

We thank the anonymous referee for useful comments.

\section{Preliminaries}

2.1. The curves $C_{t, k}$. For each locally Cohen-Macaulay curve $C \subset \mathbb{P}^{3}$ the index of speciality $e(C)$ of $C$ is the maximal integer $e$ such that $h^{1}\left(\mathcal{O}_{C}(e)\right) \neq 0$.

Fix an integer $s>0$. Let $C_{s} \subset \mathbb{P}^{3}$ be any curve fitting in an exact sequence

$$
0 \rightarrow \mathcal{O}_{\mathbb{P}^{3}}(-s-1) \rightarrow(s+1) \mathcal{O}_{\mathbb{P}^{3}}(-s) \rightarrow \mathcal{I}_{C_{s}} \rightarrow 0
$$

Each $C_{s}$ is arithmetically Cohen-Macaulay and in particular $h^{0}\left(\mathcal{O}_{C_{s}}\right)=1$. By taking the Hilbert function in (3) we get $\operatorname{deg}\left(C_{s}\right)=s(s+1) / 2, p_{a}\left(C_{s}\right)=s(s+$ $1)(2 s-5) / 6+1$ and $e\left(C_{s}\right)=s-3$. Hence $h^{i}\left(\mathcal{I}_{C_{s}}(s-1)\right)=0, i=0,1,2$. By taking $d:=\operatorname{deg}\left(C_{s}\right)$ we get $p_{a}\left(C_{s}\right)=1+d(s-1)-\left(\begin{array}{c}s+2 \\ 3\end{array}\right)=G_{A}(d, s)$. The set of all curves fitting in (3) is an irreducible variety and its general member is smooth and connected. Among them there are the stick-figures called $\mathbf{K}_{s}$ in [13], 14] and [4]. We have $h^{1}\left(N_{C_{s}}(-2)\right)=0$ for all $C_{s}$ ([11, Lemme 1], see also [10]). Unless otherwise stated we only use smooth $C_{s}$.

For any $t, k$ let $C_{t, k}:=C_{t} \sqcup C_{k}$ be the union of a smooth $C_{t}$ and a smooth $C_{k}$ with the only restriction that they are disjoint. By definition each $C_{t, k}$ is smooth. Let $d_{t, k}:=\operatorname{deg}\left(C_{t, k}\right)=t(t+1) / 2+k(k+1) / 2$ and $g_{t, k}:=h^{1}\left(\mathcal{O}_{C_{t, k}}\right)=$ $2+t(t+1)(2 t-5) / 6+k(k+1)(2 k-5) / 6$ for $t \geq k>0$. If $t \geq k>0$ then we have

$$
(t+k-1) d_{t, k}+2-g_{t, k}=\left(\begin{array}{c}
t+k+2 \\
3
\end{array}\right)
$$

Since each connected component $A$ of $C_{t, k}$ satisfies $h^{i}\left(N_{A}(-2)\right)=0, i=0,1$, we have $h^{i}\left(N_{C_{t, k}}(-2)\right)=0, i=0,1$.

Lemma 1. We have $h^{i}\left(\mathcal{I}_{C_{t, k}}(t+k-1)\right)=0, i=0,1,2$.

Proof. Since $C_{t} \cap C_{k}=\emptyset$, we have $\operatorname{Tor}_{\mathcal{O}^{3} 3}^{1}\left(\mathcal{I}_{C_{t}}, \mathcal{I}_{C_{k}}\right)=0$ and $\mathcal{I}_{C_{t}} \otimes \mathcal{I}_{C_{k}}=\mathcal{I}_{C_{t, k}}$. Therefore tensoring (3) with $s:=t$ by $\mathcal{I}_{C_{k}}(t+k-1)$ we get

$$
0 \rightarrow t \mathcal{I}_{C_{k}}(k-2) \rightarrow(t+1) \mathcal{I}_{C_{k}}(k-1) \rightarrow \mathcal{I}_{C_{t, k}}(t+k-1) \rightarrow 0
$$

We have $h^{2}\left(\mathcal{I}_{C_{k}}(k-2)\right)=h^{1}\left(\mathcal{O}_{C_{k}}(k-2)\right)$ and the latter integer is zero, because $e\left(C_{k}\right)=k-3<k-2$. We have $h^{1}\left(\mathcal{I}_{C_{k}}(k-1)\right)=0$, because $C_{k}$ is arithmetically Cohen-Macaulay. We have $h^{0}\left(\mathcal{I}_{C_{k}}(k-1)\right)=0$, by the case $s=k$ of (3). Hence $h^{i}\left(\mathcal{I}_{C_{t, k}}(t+k-1)\right)=0, i=0,1,2$.

Remark 1. In this paper we only need $k \in\{t-1, t\}$. 
Remark 2. We have $e\left(C_{t, k}\right)=\max \left\{e\left(C_{t}\right), e\left(C_{k}\right)\right\}=\max \{t-3, k-3\} \leq t+k-4$. Recall that $d_{t, k}=\operatorname{deg}\left(C_{t, k}\right)$. If $s:=t+k$, then $d_{s-1,1}=\left(s^{2}-s+2\right) / 2 \geq d_{t, k}$. If $s$ is even then $d_{t, k} \geq s(s+2) / 4=d_{\frac{s}{2}}, \frac{s}{2}$. If $s$ is odd, then $d_{t, k} \geq(s+1)^{2} / 4=d_{\frac{s+1}{2}, \frac{s-1}{2}}$.

Remark 3. Let $X$ be a general smooth curve of genus $g$ and degree $d \geq g+3$ such that $h^{1}\left(\mathcal{O}_{X}(1)\right)=0$; if either $g \geq 26\left(\left[29\right.\right.$, p. 67, inequality $\left.\left.D_{P}(g) \leq g+3\right]\right)$ or $g \leq 25$ and $d \geq g+14$ ([29, p. 67]), then $h^{1}\left(N_{X}(-2)\right)=0$ (29] uses the case $g=0$ done in 9 ).

2.2. Smoothing. We are going to apply standard smoothing techniques (see for instance [18] and 30]).

Lemma 2. Fix $A \sqcup B$ with $A=C_{t}$ and $B=C_{k}$. Let $X$ be a nodal curve with $X=A \cup B \cup Y, Y$ a smooth curve of degree $d^{\prime} \geq 2$ and genus $g^{\prime}, \sharp(A \cap Y)=1$, $\sharp(B \cap Y)=1, h^{1}\left(\mathcal{O}_{Y}(1)\right)=0$ and $h^{1}\left(N_{Y}(-2)\right)=0$. Then $h^{1}\left(N_{X}(-1)\right)=0$ and $X$ is smoothable.

Proof. Set $C:=A \cup B$. Write $\left\{p_{1}\right\}=A \cap Y$ and $\left\{p_{2}\right\}=B \cap Y$. We have an exact sequence

$$
\left.\left.\left.0 \rightarrow N_{X}(-1) \rightarrow N_{X}(-1)\right|_{C} \oplus N_{X}(-1)\right|_{Y} \rightarrow N_{X}(-1)\right|_{\left\{p_{1}, p_{2}\right\}} \rightarrow 0
$$

Since $\left.N_{X}(-1)\right|_{C}$ is obtained from $N_{C}(-1)$ by making two positive elementary transformations and $h^{1}\left(N_{C}(-1)\right)=0$, we have $h^{1}\left(\left.N_{X}(-1)\right|_{C}\right)=0$. Since $\left.N_{X}(-2)\right|_{Y}$ is obtained from $N_{Y}(-2)$ by making two positive elementary transformations and $h^{1}\left(N_{Y}(-2)\right)=0$, we have $h^{1}\left(\left.N_{X}(-2)\right|_{Y}\right)=0$. Let $H \subset \mathbb{P}^{3}$ be a general plane containing $\left\{p_{1}, p_{2}\right\}$. Since $Y$ is not a line, $Y \cap H$ is a zero-dimensional scheme. Since $h^{1}\left(\left.N_{X}(-2)\right|_{Y}\right)=0$, the restriction map

$$
H^{0}\left(Y,\left.N_{X}(-1)\right|_{Y}\right) \rightarrow H^{0}\left(Y \cap H,\left.N_{X}(-1)\right|_{H \cap Y}\right)
$$

is surjective. Since $\left\{p_{1}, p_{2}\right\} \subseteq Y \cap H$, the restriction map $H^{0}\left(Y \cap H,\left.N_{X}(-1)\right|_{H \cap Y}\right) \rightarrow$ $H^{0}\left(\left\{p_{1}, p_{2}\right\},\left.N_{X}(-1)\right|_{\left\{p_{1}, p_{2}\right\}}\right)$ is surjective. Hence the restriction map

$$
H^{0}\left(Y,\left.N_{X}(-1)\right|_{Y}\right) \rightarrow H^{0}\left(\left\{p_{1}, p_{2}\right\},\left.N_{X}(-1)\right|_{\left\{p_{1}, p_{2}\right\}}\right)
$$

is surjective. From (6) we get $h^{1}\left(N_{X}(-1)\right)=0$.

Since $h^{1}\left(N_{X}(-1)\right)=0, X$ is smoothable $([13$, Corollary 1.2]).

Call $U\left(t, k, d^{\prime}, g^{\prime}\right)$ the set of all curves $X=A \cup B \cup Y$ appearing in Lemma 2 For all integer $y \geq 0$ and $x \geq y+3$ the Hilbert scheme of smooth space curves of degree $x$ and genus $y$ is irreducible $(7,8)$. By Lemma 2 there is a unique irreducible component $W\left(t, k, d^{\prime}, g^{\prime}\right)$ of the Hilbert scheme of $\mathbb{P}^{3}$ containing the curve $X$ of Lemma 2, A general $C \in W\left(t, k, d^{\prime}, g^{\prime}\right)$ is smooth and $h^{1}\left(N_{C}(-1)\right)=0$. We have $\operatorname{deg}(C)=d^{\prime}+\operatorname{deg}\left(C_{t}\right)+\operatorname{deg}\left(C_{k}\right)=d^{\prime}+t(t+1) / 2+k(k+1) / 2$ and genus $g(C)=g^{\prime}+p_{a}\left(C_{t}\right)+p_{a}\left(C_{k}\right)=g^{\prime}-2+t(t+1)(2 t-5) / 6+k(k+1)(2 k-5) / 6$.

\section{Assertion $M(s, t, k), k \in\{t-1, t\}$}

For any $t \geq 27$, set $c(2 t+1, t, t)=t+3, d(2 t+1, t, t)=0, c(2 t, t, t-1)=t+2$ and $d(2 t, t, t-1)=t-1$. Set $g(t+k+1, t, k):=c(t+k+1, t, k)-3$. Note that if $k \in\{t-1, t\}$ we have

$$
t(t+1)+k(k+1)+d(t+k+1, t, k)=(t+k)(t+k+4-c(t+k+1, t, k))
$$


Now fix an integer $s \geq t+k+3$ with $s-t-k-1 \equiv 0(\bmod 2)$ and define the integers $c(s, t, k), g(s, t, k)$ and $d(s, t, k)$ in the following way. Set

$$
\begin{gathered}
c(s, t, k):=\left\lfloor\frac{\left(\begin{array}{c}
s+3 \\
3
\end{array}\right)-s d_{t, k}-6-3(s-t-k-1) / 2+g_{t, k}}{s-1}\right\rfloor, \\
d(s, t, k):=\left(\begin{array}{c}
s+3 \\
3
\end{array}\right)-s d_{t, k}-6-3(s-t-k-1) / 2+g_{t, k}-(s-1) c(s, t, k),
\end{gathered}
$$

and $g(s, t, k):=c(s, t, k)-3-3(s-t-k-1) / 2$. Note that

(8) $s\left(d_{t, k}+c(s, t, k)\right)+3-g_{t, k}-g(s, t, k)+d(s, t, k)=\left(\begin{array}{c}s+3 \\ 3\end{array}\right), 0 \leq d(s, t, k) \leq s-2$

and (8) holds even if $s=t+k+1$. From (8) for the integers $s+2$ and $s$ and the equality $g(s+2, t, k)-g(s, t, k)=c(s+2, t, k)-c(s, t, k)-3$ we get

$$
\begin{aligned}
& 2 d_{t, k}+2 c(s, t, k)+(s+1)(c(s+2, t, k)-c(s, t, k))+ \\
& d(s+2, t, k)-d(s, t, k)+3=(s+3)^{2}
\end{aligned}
$$

Remark 4. We have $c(2 t+1, t, t)=t+3, d(2 t+1, t, t)=0, c(2 t, t, t-1)=t+2$, $d(2 t, t, t-1)=t-1, c(2 t+2, t, t-1)=2 t+6, d(2 t+2, t, t-1)=2 t-3$, $c(2 t+3, t, t)=2 t+7, d(2 t+3, t, t)=2 t-1$.

Remark 5. We explain here the main reason for the assumption $t \geq 27$ made in this section. Fix an integer $s \geq t+k+1$ with $s \equiv t+k+1(\bmod 2)$. We work with a curve $X=C_{t, k} \sqcup A$ with $A$ a general smooth curve of degree $c(s, t, k)$ and genus $g(s, t, k)$ and we need $h^{1}\left(N_{X}(-2)\right)=0$, i.e. we need $h^{1}\left(N_{A}(-2)\right)=0$. We have $c(s, t, k) \geq g(s, t, k)+3$. By Lemma 4 below we have $g(s, t, k) \geq g(t+k+1, t, k)$. We have $g(2 t+1, t, t)=t \geq 27$ and $g(2 t, t, t-1)=t-1 \geq 26$. Since $g(s, t, k) \geq 26$, Remark 3 gives $h^{1}\left(N_{A}(-2)\right)=0$.

Lemma 3. For all integer $s \geq t+k+1$ with $s \equiv t+k-1(\bmod 2)$ and $t \geq 27$ we have $g_{\lceil(s+1) / 2\rceil,\lfloor(s+1) / 2\rfloor}>g_{t, k}+g(s, t, k)$.

Proof. The lemma is true if $s=t+k+1$ by the explicit value of $g(t+k+$ $1, t, k)=c(t+k+1, t, k)-3$ (Remark 4). Now let $s \geq t+k+3$ and assume that the lemma is true for the integer $s-2$. Since $s-2 \geq t+k+1$ the inductive assumption gives $g_{\lceil(s-1) / 2\rceil,\lfloor(s-1) / 2\rfloor}>g_{t, k}+g(s-2, t, k)$. Thus it is sufficient to check that $g_{\lceil(s+1) / 2\rceil,\lfloor(s+1) / 2\rfloor}-g_{\lceil(s-1) / 2\rceil,\lfloor(s-1) / 2\rfloor} \geq g(s, t, k)-g(s-2, t, k)=$ $c(s, t, k)-c(s-2, t, k)-3$. An elementary numerical computation shows that this inequality holds for any $s>t \geq 27$ : indeed, the key point is that the difference $g_{\lceil(s+1) / 2\rceil,\lfloor(s+1) / 2\rfloor}-g_{\lceil(s-1) / 2\rceil,\lfloor(s-1) / 2\rfloor}$ is quadratic in $s$ by definition of $g_{t, k}$, while the difference $c(s, t, k)-c(s-2, t, k)$ is linear in $s$ by (9).

Lemma 4. For each $s \geq t+k+1$ with $s \equiv t+k-1(\bmod 2)$ we have $2(c(s+$ $2, t, k)-c(s, t, k)) \geq s+4$.

Proof. Since $g_{t, k}+g(s, t, k)<g_{\lceil(s+1) / 2\rceil,\lfloor(s+1) / 2\rfloor}$ (Lemma 3), (8) for $s, t, k$ and (1) for $t^{\prime}=\lceil(s+1) / 2\rceil$ and $k^{\prime}=\lfloor(s+1) / 2\rfloor$ imply $d_{t^{\prime}, k^{\prime}} \geq c(s, t, k)+d_{t, k}$. Remark 4 gives $c\left(s+2, t^{\prime}, k^{\prime}\right)=k^{\prime}+3$. Since $0 \leq d(s+2, t, k) \leq s$ and $0 \leq d(s, t, k) \leq$ $s-2$, (9) and the difference between (8) for $s^{\prime}:=s+2$ and (4) for $t^{\prime}, k^{\prime}$ imply $c(s+2, t, k)-c(s, t, k) \geq-1+c\left(s+2, t^{\prime}, k^{\prime}\right)=\lfloor(s+1) / 2\rfloor+2$. 
Let $Q:=\mathbb{P}^{1} \times \mathbb{P}^{1}$. The elements of $\left|\mathcal{O}_{Q}(0,1)\right|$ are the fibers of the projection $\pi_{2}: Q \rightarrow \mathbb{P}^{1}$, so that each $D \in\left|\mathcal{O}_{Q}(1,0)\right|$ contains exactly one point of each fiber of $\pi_{2}$.

Assertion $M(s, t, k), k \in\{t-1, t\}, s \geq t+k+1, s \equiv t+k+1(\bmod 2)$ : Set $e=1$ if $0 \leq d(s, t, k) \leq c(s+2, t, k)-c(s, t, k)-3$ and $e=2$ if $d(s, t, k)>$ $c(s+2, t, k)-c(s, t, k)-3$. There is a 6 -tuple $\left(X, Q, D_{1}, D_{2}, S_{1}, S_{2}\right)$ such that

(a) $Q$ is a smooth quadric surface, $X=C_{t, k} \sqcup Y, Y$ is a smooth curve of degree $c(s, t, k)$ and genus $g(s, t, k)$ and $Q$ intersects transversally $X$, with no line of $Q$ containing $\geq 2$ points of $X \cap Q$;

(b) $D_{1}, D_{2}$ are different elements of $\left|\mathcal{O}_{Q}(1,0)\right|$, each of them containing one point of $Y \cap Q, S_{i} \subset D_{i} \backslash D_{i} \cap Y, 1 \leq i \leq 2$, and $\sharp\left(S_{1}\right)+\sharp\left(S_{2}\right)=d(s, t, k)$; $\pi_{2}\left(S_{2}\right) \subseteq \pi_{2}\left(S_{1}\right) ; S_{2}=\emptyset$ and $\pi_{2}\left(S_{1}\right) \subseteq \pi_{2}\left(Y \cap\left(Q \backslash\left(D_{1} \cup D_{2}\right)\right)\right)$ if $e=1$, $\sharp\left(S_{2}\right)=d(s, t, k)-c(s+2, t, k)+c(s, t, k)+3$ and $\pi_{2}\left(S_{2}\right) \subseteq \pi_{2}(Y \cap(Q)$ $\left.\left.\left(D_{1} \cup D_{2}\right)\right)\right)$ if $e=2$;

(c) $h^{i}\left(\mathcal{I}_{X \cup S_{1} \cup S_{2}}(s)\right)=0, i=0,1$.

Remark 6. Fix lines $L, R \subset \mathbb{P}^{3}$ such that $L \cap R=\emptyset$ and $o \in \mathbb{P}^{3} \backslash(L \cup R)$. Let $\ell: \mathbb{P}^{3} \backslash\{o\} \rightarrow \mathbb{P}^{2}$ denote the linear projection from $o$. We have $\sharp(\ell(L) \cap \ell(R))=1$, i.e. there is a unique line $D(L, R, o) \subset \mathbb{P}^{3}$ such that $o \in D(L, R, o), D(L, R, o) \cap L \neq \emptyset$ and $D(L, R, o) \cap R \neq \emptyset$. We have $\sharp(D(L, R, o) \cap L)=\sharp(D(L, R, o) \cap R)=1$. The function $(L, R, o) \mapsto D(L, R, o)$ is regular.

Remark 7. For any $o \in \mathbb{P}^{3}$ let $\chi(o)$ denote the first infinitesimal neighbourhood of $o$ in $\mathbb{P}^{3}$, i.e. the closed subscheme of $\mathbb{P}^{3}$ with $\mathcal{I}_{o}^{2}$ as its ideal sheaf. For any surface $F \subset \mathbb{P}^{3}$ and any scheme $B \subset \mathbb{P}^{3}$ let $\operatorname{Res}_{F}(B)$ denote the closed subscheme of $\mathbb{P}^{3}$ with $\mathcal{I}_{B}: \mathcal{I}_{F}$ as its ideal sheaf. We have $\operatorname{Res}_{F}(B) \subseteq B$. If $B$ is the disjoint union of closed subschemes $B_{1}$ and $B_{2}$ then $\operatorname{Res}_{F}(B)=\operatorname{Res}_{F}\left(B_{1}\right) \cup \operatorname{Res}_{F}\left(B_{2}\right)$. If $B$ is reduced then $\operatorname{Res}_{F}(B)$ is the union of the irreducible components of $B$ not contained in $F$. If $o \notin F$ then $\operatorname{Res}_{F}(\chi(o))=\chi(o)$. If $o \in F$ and $F$ is smooth at $o$ then $\operatorname{Res}_{F}(\chi(o))=\{o\}$.

Lemma 5. For all $t \geq 27$ and $k \in\{t-1, t\}$ assertion $M(t+k+1, t, k)$ is true.

Proof. Fix $C_{t, k}$ intersecting $Q$ at $2 d_{t, k}$ general points (29).

(a) Assume $k=t$. We have $c(2 t+1, t, t)=t+3$ and $d(2 t+1, t, t)=0$ and so we take $e=1$ with $S_{1}=S_{2}=\emptyset$. Take any $A \in\left|\mathcal{O}_{Q}(2, t+1)\right|$ with $A \cap C_{t, k}=\emptyset$. We have $\operatorname{Res}_{Q}\left(C_{t, t} \cup A\right)=C_{t, t}$ and thus $h^{i}\left(\mathcal{I}_{\operatorname{Res}_{Q}\left(C_{t, t} \cup A\right)}(2 t-1)\right)=0, i=0,1$. We have $h^{i}\left(Q, \mathcal{I}_{Q \cap(C \cap A)}(2 t+1,2 t+1)\right)=h^{i}\left(Q, \mathcal{I}_{C_{t, t} \cap Q}(2 t-1, t)\right)=0, i=0,1$, by (7) and the generality of $C_{t, k} \cap Q$. Hence $h^{i}\left(\mathcal{I}_{C_{t, k} \cup A}(2 t+1)\right)=0, i=0,1$.

We deform $A$ to a curve $Y$ of degree $t+3$ and genus $t$ with $Y \cap C_{t, k}=\emptyset$, $Y$ intersecting transversally $Q$ and with no line of $Q$ containing $\geq 2$ points of $Q \cap\left(C_{t, k} \cup Y\right)$. By the semicontinuity theorem for cohomology ([15, III.8.8]), for a general $Y$ we have $h^{i}\left(\mathcal{I}_{C_{t, k} \cup Y}(2 t+1)\right)=0, i=0,1$. Set $X:=C_{t, k} \cup Y, S_{1}=S_{2}=\emptyset$ and take as $D_{1}$ and $D_{2}$ any two different elements of $\left|\mathcal{O}_{Q}(1,0)\right|$, each of them containing one point of $Y \cap Q$.

(b) Assume $k=t-1$. We have $c(2 t, t, t-1)=t+2, d(2 t, t, t-1)=t-1$ and $c(2 t+2, t, t-1)-c(2 t, t, t-1)=t+4$ (Remark 44). Hence $e=1$. However, in the proof of $M(t+k+1, t, k)$ we will exchange the two rulings (as we will do below for the general proof that $M(s, t, k) \Longrightarrow M(s+2, t, k))$, so that $D_{1}, D_{2} \in\left|\mathcal{O}_{Q}(0,1)\right|$. Take lines $L_{1}, L_{2} \in\left|\mathcal{O}_{Q}(1,0)\right|$ such that $L_{1} \neq L_{2}$ and $C_{t, t-1} \cap\left(L_{1} \cup L_{2}\right)=\emptyset$, and 
$t$ different lines $R_{j} \in\left|\mathcal{O}_{Q}(0,1)\right|, 1 \leq j \leq t$, none of them containing a point of $C_{t, t-1} \cap Q$. Fix $D_{1}, D_{2} \in\left|\mathcal{O}_{Q}(0,1)\right|$ containing no point of $C_{t, t-1} \cap Q$ and with $D_{h} \neq R_{j}$ for all $h, j$. Set $u_{h}:=L_{1} \cap D_{h}, h=1,2$. Fix $E_{1} \subset D_{1}$ with $\sharp\left(E_{1}\right)=t-1$ and $E_{1} \cap\left(L_{1} \cup L_{2}\right)=\emptyset$. We have $h^{1}\left(Q, \mathcal{I}_{E_{1}}(2 t-2, t)\right)=0$. Since $C_{t, k} \cap Q$ is a general subset of $Q$ with cardinality $2 d_{t, k}$, we have $h^{i}\left(Q, \mathcal{I}_{Q \cap(C \cap A) \cup E_{1}}(2 t, 2 t)\right)=$ $h^{i}\left(Q, \mathcal{I}_{\left(C_{t, t} \cap Q\right) \cup E_{1}}(2 t-2, t)\right)=0, i=0,1$, by (7). The residual sequence of $Q$ gives $h^{i}\left(\mathcal{I}_{C_{t, k}} \cup A \cup E_{1}(2 t)\right)=0, i=0,1$.

Take an ordering $\left\{o_{1}, \ldots, o_{t-1}\right\}$ of $E_{1}$ and let $M_{i}$ the only element of $\left|\mathcal{O}_{Q}(1,0)\right|$ with $o_{i} \in M_{i}$. Set $w_{i}:=R_{i} \cap M_{i}, 1 \leq i \leq t-1$. We fix a deformation $\left\{L_{h}(\lambda)\right\}_{\lambda \in \Lambda}$, $h=1,2$, of $L_{h}$ with the following properties: $\Lambda$ is a connected and affine smooth curve, $o \in \Lambda, L_{h}(o)=L_{h}, u_{h} \in L_{h}(\lambda)$ for all $\lambda, L_{1}(\lambda) \cap L_{2}(\lambda)=\emptyset$ for all $\lambda$ and $L_{h}(\lambda)$ is transversal to $Q$ for all $\lambda \neq o$. For each $i$ with $1 \leq i \leq t-1$ there is a unique line $R_{i}(\lambda)$ containing $w_{i}$ and intersecting both $L_{1}(\lambda)$ and $L_{2}(\lambda)$ (Remark 6). There is a deformation $\left\{R_{t}(\lambda)\right\}_{\lambda \in \Lambda}$ of $R_{t}$ with $R_{t}(o)=R_{t}, R_{t}(\lambda)$ intersecting both $L_{1}(\lambda)$ and $L_{2}(\lambda)$. Taking instead of $\Lambda$ a smaller neighborhood of $o$ we may assume $R_{i}(\lambda) \cap R_{j}(\lambda)=\emptyset$ for all $i \neq j$ and all $\lambda$ so that $A(\lambda):=$ $L_{1}(\lambda) \cup L_{2}(\lambda) \cup R_{1}(\lambda) \cup \cdots \cup R_{t}(\lambda)$ is a connected nodal curve of degree $t+2$ and arithmetic genus $t-1$. By semicontinuity (restricting if necessary $\Lambda$ to a neighborhood of $o$ ) we have $h^{i}\left(\mathcal{I}_{C_{t, k} \cup A(\lambda) \cup E_{1}}(2 t)\right)=0, i=0,1$, for all $\lambda \in \Lambda$. Fix $\lambda_{0} \in \Lambda \backslash\{o\}$. Let $\left\{B_{\delta}\right\}_{\delta \in \Delta}$ be a smoothing of $A\left(\lambda_{0}\right)$ fixing $u_{1}$ and $u_{2}$, i.e. take a smooth and connected affine curve $\Delta$ and $a \in \Delta$ with $B_{a}=A\left(\lambda_{0}\right), B_{\delta}$ a smooth curve of degree $t+2$ and genus $t-1$ and $\left\{u_{1}, u_{2}\right\} \subset B_{\delta}$ for all $\delta$. Restricting if necessary $\Delta$ we may assume that $B_{\delta}$ is transversal to $Q$ and disjoint from $C_{t, k} \cup E_{1}$ for all $\delta \in \Delta$ and (by semicontinuity) that $h^{i}\left(\mathcal{I}_{C_{t, k} \cup B_{\delta} \cup E_{1}}(2 t)\right)=0, i=0,1$. Since $A\left(\lambda_{0}\right)$ is transversal to $Q$, we may (up to a finite covering of $\Delta$ ) find $t-1$ sections $s_{1}, \ldots, s_{t-1}$ of the family $\left\{B_{\delta} \cap Q\right\}_{\delta \in \Delta}$ of $2 t+4$ ordered points of $Q$ with $s_{i}(a)=w_{i}, i=1, \ldots, t-1$. Let $M_{j}(\delta), \delta \in \Delta$, be the only element of $\left|\mathcal{O}_{Q}(1,0)\right|$ with $w_{i} \in M_{i}(\delta)$. Set $o_{i}(\delta):=L_{1} \cap M_{i}(\delta)$ and $E_{1}(\delta):=\left\{o_{1}(\delta), \ldots, o_{t-1}(\delta)\right\}$. By semicontinuity for a general $\delta \in \Delta \backslash\{a\}$ we have $h^{i}\left(\mathcal{I}_{C_{t, k} \cup B_{\delta} \cup E_{1}(\delta)}(2 t)\right)=0$. We fix such a $\delta$ and set $X:=C_{t, k} \cup B_{\delta}, S_{1}:=E_{1}(\delta), S_{2}:=\emptyset$. For $M(2 t, t, t-1)$ we use the lines $D_{1}, D_{2}$ and $M_{j}(\delta), 1 \leq j \leq t-1$.

Lemma 6. For each integer $s \geq t+k+1$ such that $s \equiv t+k+1(\bmod 2)$ we have $2 c(s, t, k) \geq s+4$ and $2 c(s, t, k) \geq s+6$ is $s \geq t+k+3$.

Proof. The case $s=t+k+1$ is true by Remark 4 . The general case follows by induction $s-2 \Longrightarrow s$ by Lemma 4 .

We need the following auxiliary result, proved in [5, Lemma 2.5] and [17, bottom of page 176].

Lemma 7. Fix lines $D, L \subset \mathbb{P}^{3}$ such that $D \cap L$ is a point o and $q \in L \backslash\{o\}$. Then there is a family $\left\{L_{\lambda}\right\}_{\lambda \in \mathbb{K}}$ of lines of $\mathbb{P}^{3}$ such that $L_{0}=L, L_{\lambda} \cap D=\emptyset$ for all $t \neq 0$, $D \cup L \cup \chi(o)$ is a flat limit of the family $\left\{D \cup L_{\lambda}\right\}_{\lambda \in \mathbb{K} \backslash\{0\}}$.

Proof. Take homogenous coordinates $x_{0}, x_{1}, x_{2}, x_{3}$ such that $o=(1: 0: 0: 0)$, $D=\left\{x_{1}=x_{2}=0\right\}, L=\left\{x_{1}=x_{3}=0\right\}$ and $q=(0: 0: 1: 0)$. Take $L_{\lambda}=\left\{x_{1}+\lambda x_{0}=x_{3}=0\right\}$. Note that $L_{0}=L$ and that $L \cap D=\emptyset$ for all $\lambda \neq 0$. Set $Y_{\lambda}:=D \cup L_{\lambda}$. For $\lambda \neq 0$ the ideal sheaf of scheme $Y_{t}$ is generated by the quadrics $x_{1}\left(x_{1}+\lambda x_{0}\right), x_{2}\left(x_{1}+\lambda x_{0}\right), x_{1} x_{3}, x_{2} x_{3}$, while the ideal sheaf of the scheme $Y_{0}$ is determined by the quadrics $x_{1}^{2}, x_{1} x_{2}, x_{1} x_{3}$ and $x_{2} x_{3}$. This algebraic 
family of projective schemes is flat because it has constant Hilbert polynomial [15], III.9.8.4].

Lemma 8. Assume $t \geq 27$ and $k \in\{t-1, t\}$. Fix an integer $s \geq t+k+1$ such that $s \equiv t+k+1(\bmod 2)$. If $M(s, t, k)$ is true, then $M(s+2, t, k)$ is true.

Proof. Let $e \in\{1,2\}$ be the integer arising in $M(s, t, k)$ and $f \in\{1,2\}$ the corresponding integer for $M(s+2, t, k)$. Take $\left(X, Q, D_{1}, D_{2}, S_{1}, S_{2}\right)$ satisfying $M(s, t, k)$ with $X=C_{t, k} \sqcup Y$ and $D_{1}, D_{2} \in\left|\mathcal{O}_{Q}(1,0)\right|$. The 6 -tuple $\left(X^{\prime}, Q, D_{1}^{\prime}, D_{2}^{\prime}, S_{1}^{\prime}, S_{2}^{\prime}\right)$ will be a solution after exchanging the two rulings of $Q$, i.e. we will take $D_{1}^{\prime}, D_{2}^{\prime} \in$ $\left|\mathcal{O}_{Q}(0,1)\right|$ and we use $\pi_{1}$ instead of $\pi_{2}$. In each step with $d(s, t, k) \neq 0$ we obtain $X^{\prime}$ smoothing a curve $W$ union of $X, \chi:=\cup_{o \in S_{1} \cup S_{2}} \chi(o), e+1$ elements $\left|\mathcal{O}_{Q}(1,0)\right|$ and $c(s+2, t, k)-c(s, t, k)-e-1$ elements of $\left|\mathcal{O}_{Q}(0,1)\right|$. See step (c) for the easier case $d(s, t, k)=0$ (here to get $W$ we add to $X$ a line $D_{0} \in\left|\mathcal{O}_{Q}(1,0)\right|$ and $c(s+2, t, k)-c(s, t, k)-1$ elements of $\left.\left|\mathcal{O}_{Q}(0,1)\right|\right)$.

(a) Assume $e=2$ and set $z:=d(s, t, k)+3-c(s+2, t, k)+c(s, t, k)$. Since $d(s, t, k) \leq s-2$, Lemma 4 gives $d(s, t, k) \leq 2(c(s+2, t, k)-c(s, t, k)-3)$, i.e. $z \leq c(s+2, t, k)-c(s, t, k)-3$. By assumption there is $E \subset Y \cap\left(Q \backslash\left(D_{1} \cup D_{2}\right)\right)$ such that $\sharp(E)=z$ and $\pi_{2}(E)=\pi_{2}\left(S_{2}\right) \subseteq \pi_{2}\left(S_{1}\right)$. Take a line $D_{0} \in\left|\mathcal{O}_{Q}(1,0)\right|$ different from $D_{1}, D_{2}$, with $D_{0} \cap E=\emptyset, D_{0} \cap C_{t, k} \cap Q=\emptyset$ and $D_{0} \cap Y \cap Q \neq \emptyset$; we use that $2 c(s, t, k) \geq 3+z$ (Lemma 6 ). Take distinct lines $L_{i} \in\left|\mathcal{O}_{Q}(0,1)\right|$, $1 \leq i \leq c(s+2, t, k)-c(s, t, k)-3$, such that $L_{i} \cap Y \neq \emptyset$ if and only if $i \leq z$, $X \cap\left(\bigcup_{i=1}^{c(s+2, t, k)-c(s, t, k)-3} L_{i}\right)=E, L_{i} \cap\left(C_{t, k} \cap Q\right)=\emptyset$ for all $i$. Set $J:=\left(D_{0} \cup D_{1} \cup\right.$ $\left.D_{2}\right) \cup\left(\bigcup_{i=1}^{c(s+2, t, k)-c(s, t, k)-3} L_{i}\right)$. We fix $f$ general lines $R_{i} \in\left|\mathcal{O}_{Q}(0,1)\right|, 1 \leq i \leq f$, and $A_{i} \subset R_{i}, 1 \leq i \leq f$, with the conditions $\sum_{i=1}^{f} \sharp\left(A_{i}\right)=b(s+2, t, k), \pi_{1}\left(A_{f}\right) \subseteq$ $\pi_{1}\left(A_{1}\right)$ and $\pi_{1}\left(A_{f}\right) \subseteq \pi_{1}(Y \cap(Q \backslash J))$. Set $\chi:=\cup_{o \in S_{1} \cup S_{2}} \chi(o), A:=A_{1} \cup A_{2}$ and $W:=X \cup J \cup \chi$.

Claim 1: There is an affine connected smooth curve $\Delta$ such that the scheme $Y \cup J \cup \chi$ is a flat degeneration of a family of unions of $Y \cup D_{0} \cup D_{1} \cup D_{2}$ and $c(s+2, t, k)-c(s, t, k)-3$ lines $L_{i \lambda}, \lambda \in \Delta$, such that $L_{i \lambda} \cap Y \neq \emptyset$ if and only if $i \leq z, D_{0} \cap L_{i \lambda}=D_{0} \cap L_{i}$ for all $i, D_{1} \cap L_{i \lambda}=\emptyset$ for all $\lambda \in \Delta$ and all $i$, and $D_{2} \cap L_{i \lambda} \neq \emptyset$ (and it is a point) if and only if $z+1 \leq i \leq c(s+2, t, k)-c(s, t, k)-3$.

Proof of Claim 1: As $\Delta$ we take a suitable curve contained in the affine manifold $\prod_{i=1}^{c(s+2, t, k)-c(s, t, k)-3} \Delta_{i}$, which we are now going to define (in the use of Claim 1 we only need that $\Delta$ is irreducible, so we could use a similar claim, but with this connected manifold instead of the irreducible curve $\Delta$ ). Each $\Delta_{i}$ is a smooth connected curve, so $\prod_{i=1}^{c(s+2, t, k)-c(s, t, k)-3} \Delta_{i}$ is irreducible. We describe each $L_{i \lambda}$ with $L_{i}$ as a limit separately for each $i ; \Delta_{i}$ is the parameter space for the line $L_{i \lambda}$. We need to modify the proof of Lemma 7 in the following way. First assume $z<i \leq c(s+2, t, k)-c(s, t, k)-3$. In this case we fix the point $q_{i}:=D_{0} \cap L_{i}$ and use as $\Delta_{i}$ a Zariski open neighborhood of $D_{2} \cap L_{i}$; for each $q \in D_{2}$ there is a unique line $L\left(q_{i}, q\right)$ containing $\left\{q_{i}, q\right\}$; when $q$ goes to $D_{2} \cap L_{i}$ the line $L\left(q_{i}, q\right)$ goes to the line; we need to restrict $\Delta_{i}$ to avoid the points $q$ such that $L\left(q_{i}, q\right) \cap\left(Y \cup C_{t, k} \cup D_{1}\right) \neq \emptyset$. Now assume $i \leq z$. We fix the point $q_{i}:=Y \cap L_{i}$ and take as $\Delta_{i}$ a Zariski neighborhood of $q_{i}$ in $Y$; since $q_{i} \notin D_{0}$ for each $q \in D_{0} \backslash Y \cap D_{0}$ there is a unique line $L\left(q_{i}, q\right)$ containing $\left\{q_{i}, q\right\}$; we need to restrict $\Delta_{i}$ to avoid the points $q$ such that $L\left(q_{i}, q\right) \cap\left(Y \cup C_{t, k}\right) \neq\{q\}$. We restrict $\Delta_{1} \times \cdots \times \Delta_{c(s+2, t, k)-c(s, t, k)-3}$ to a nonempty Zariski open subset $U$ such that for all $\lambda \in U$ the union $U^{\prime}$ of $Y \cup D_{0} \cup D_{1} \cup D_{2}$ and the line $L_{i \lambda}, 1 \leq i \leq c(s+2, t, k)-c(s, t, k)-3$ is nodal and it has no singular 
point which is not prescribed by the construction. Note that $U^{\prime}$ is connected and $p_{a}\left(U^{\prime}\right)=g(s+2, t, k)$.

Claim 2: $W$ is a flat degeneration of a disjoint union of $C_{t, k}$ and a smooth curve of degree $c(s+2, t, k)$ and genus $g(s+2, t, k)$.

Proof of Claim 2: Since $C_{t, k} \cap(J \cap \chi)=C_{t, k} \cap Y=\emptyset$, it is sufficient to prove that $Y \cup J \cup \chi$ is a flat degeneration of a family of smooth curves of degree $c(s+2, t, k)$ and genus $g(s+2, t, k)$. By Claim $1, Y \cup J \cup \chi$ is a flat degeneration of a family of unions of $Y \cup D_{0} \cup D_{1} \cup D_{2}$ and $c(s+2, t, k)-c(s, t, k)-3$ disjoint lines, none of them intersecting $D_{1} \cup D_{2}$ and each of them intersecting $Y \cup D_{0} \cup D_{1} \cup D_{2}$ quasi transversely at exactly two points. We first prove that $Y \cup J \cup \chi$ is a flat degeneration of a family of unions of $Y \cup D_{0} \cup D_{1} \cup D_{2}$ and $c(s+2, t, k)-c(s, t, k)-3$ lines $L_{i \lambda}, \lambda \in \mathbb{K} \backslash\{0\}$, such that $L_{\cap i \lambda} \cap Y \neq \emptyset$ if and only if $i \leq z, D_{0} \cap L_{i \lambda}=D_{0} \cap L_{i}$ for all $i$ and $D_{1} \cap L_{i \lambda}=D_{2} \cap L_{i \lambda}=\emptyset$ for all $\lambda \in \mathbb{K} \backslash\{0\}$. We may do this smoothing separately, first for $D_{0}, D_{1}, D_{2}$ and then for each line $L_{i \lambda}$ quoting each time Lemma 2 and following the deformation with a family of lines with special fiber $L_{j \lambda}, j \neq i$, because any two points of $\mathbb{P}^{3}$ uniquely determine a line and the line depends regularly if we move regularly the two points (see the Side Remark below), but we may do all the smoothings simultaneously just choosing the appropriate references from [18, or [30, or other sources, e.g. Lemma 2] or [18, Corollary 5.2].

Side Remark: As the reader may have noticed in the proof Claim 1 we only used Lemma 7 (i.e. a known result) and the fact that two different points $q, q^{\prime}$ of $\mathbb{P}^{r}, r \geq 3$, uniquely determine a line $L\left(q, q^{\prime}\right) \subset \mathbb{P}^{3}$ and the regularity of the map $\left(q, q^{\prime}\right) \rightarrow L\left(q, q^{\prime}\right)$ from $\mathbb{P}^{r} \times \mathbb{P}^{r} \backslash \Delta_{\mathbb{P}^{r}}$, where $\Delta_{\mathbb{P}^{r}}$ is the diagonal, to the Grassmaniann $G(1, r)$. Lemma 7 is true in a more general situation, as the flat limit of a two smooth germs of curves colliding to an ordinary node; call $o$ this nodal point. Instead of the lines $D, L$ we take the tangent lines to the two smooth germs of curves. Their linear span determines an element of $G(3, r)$ and we consider the first infinitesimal neighborhood $\chi(o)$ of $o$ in a 3-dimensional projective space which is a limit of these elements of $G(3, r)$. In the literature Claims 1 and 2 are often used, but without separating them. For the algebraic geometers of our generation the first instance of this flat limit with a nilpotent was [15, III.9.8.4 and figure 11 at page 260].

To obtain a smoothing of $W$ as in the Claim 2, but compatible with the data $A_{1}, A_{2}$, see steps (a1) and (a2). We have $\operatorname{Res}_{Q}(W \cup A)=X \cup S_{1} \cup S_{2}$ and so $h^{i}\left(\mathcal{I}_{\operatorname{Res}_{Q}(W \cup A)}(s)\right)=0, i=0,1$. We have $h^{i}\left(Q, \mathcal{I}_{(W \cap Q) \cup A}(s+2, s+2)\right)=$ $h^{i}\left(Q, \mathcal{I}_{(X \cap(Q \backslash J) \cup A}(s-1, s+5+c(s, t, k)-c(s+2, t, k))\right)$. We have $\sharp((X \cap(Q \backslash$ $J)) \cup A)=h^{0}\left(Q, \mathcal{O}_{Q}(s-1, s+5+c(s, t, k)-c(s+2, t, k))\right.$. We have $h^{1}\left(Q, \mathcal{I}_{A}(s-\right.$ $1, s+5+c(s, t, k)-c(s+2, t, k)))=0$, because $s+5+c(s, t, k)-c(s+2, t, k)>0$, $f \leq 2$ and $\sharp\left(A_{1}\right) \leq s$; this is a key reason for our definition of $M(s+2, t, k)$. Therefore to prove that $h^{i}\left(Q, \mathcal{I}_{(X \cap(Q \backslash J) \cup A}(s-1, s+5+c(s, t, k)-c(s+2, t, k))\right)=0$, $i=0,1$, it is sufficient to prove that we may take as $X \cap(Q \backslash J)$ a general subset of $Q$ with its prescribed cardinality. By Remark 5 we have $h^{1}\left(N_{X}(-2)\right)=0$. Since $h^{1}\left(N_{X}(-2)\right)=0$, we may deform $X$ keeping fixed $E$ so that the other points are general in $Q$.

(a1) We have just proved that $h^{i}\left(\mathcal{I}_{W \cup A}(s+2)\right)=0, i=0,1$. If $d(s+2, t, k)=0$, then $M(s+2, t, k)$ is proved for $e=2$. Now assume $d(s+2, t, k)>0$. To prove $M(s+2, t, k)$ when $e=2$ we need to deform $W$ to a smooth $X^{\prime}=C_{t, k} \sqcup Y^{\prime}$ intersecting transversally $Q$ and (perhaps moving $A$ ) to obtain condition (b) of 
$M(s+2, t, k)$. Set $P_{i}:=Y \cap D_{i}, i=0,1,2$. Let $\left\{D_{i}(\lambda)\right\}_{\lambda \in \Lambda}$ be a deformation of $D_{i}$ with $\Lambda$ a smooth and connected affine curve, $o \in \Lambda, D_{i}(o)=D_{i}, D_{i}(\lambda), \lambda \in \Lambda \backslash\{o\}$, a line of $\mathbb{P}^{3}$ transversal to $Q$ and containing $P_{i}$. Fix $i \in\{1, \ldots, z\}$. By Remark 6 for each $\lambda \in \Lambda$ there is a unique line $L_{i}(\lambda) \subset \mathbb{P}^{3}$ such that $D_{0} \cap L_{i} \in L_{i}(\lambda)$, $L_{i}(\lambda) \cap D_{1}(\lambda) \neq \emptyset$ and $L_{i}(\lambda) \cap D_{2}(\lambda) \neq \emptyset$; restricting if necessary $\Lambda$ we may assume that all $L_{i}(\lambda), \lambda \neq o$, are transversal to $Q$. Fix an integer $i$ with $z<i \leq$ $c(s+2, t, k)-c(s, t, k)-3$ and fix a general $m_{i} \in L_{i}$. By Remark 6 there is a unique line $L_{i}(\lambda)$ such that $m_{i} \in L_{i}(\lambda), L_{i}(\lambda) \cap D_{1}(\lambda) \neq \emptyset$ and $L_{i}(\lambda) \cap D_{2}(\lambda) \neq \emptyset$; restricting if necessary $\Lambda$ we may assume that all $L_{i}(\lambda), \lambda \neq o$, are transversal to $Q$. Restricting if necessary $\Lambda$ to a smaller neighborhood of $o$ in $\Lambda$ we may assume that $L_{i}(\lambda) \cap L_{j}(\lambda)=\emptyset$ for all $i \neq j$, that $C_{t, k} \cap L_{i}(\lambda)=\emptyset$ for all $i$ and all $\lambda$, that $L_{i}(\lambda) \cap D_{0} \neq \emptyset$ if and only if $i \leq z$. Fix a general $\lambda \in \Lambda$ and set $J(\lambda):=D_{0}(\lambda) \cup D_{1}(\lambda) \cup D_{2}(\lambda) \cup\left(\bigcup_{i=1}^{c(s+2, t, k)-c(s, t, k)-3} L_{i}(\lambda)\right)$. Let $\chi(\lambda)$ be the union of all $\chi(q)$ with either $q \in D_{1}(\lambda) \cap L_{i}(\lambda), 1 \leq i \leq c(s+2, t, k)-c(s, t, k)-3$ or $q \in D_{2}(\lambda) \cap L_{i}(\lambda), 1 \leq i \leq z$. Set $W(\lambda):=X \cup J(\lambda) \cup \chi(\lambda)$. $W(\lambda)$ is the disjoint union of $C_{t, k}$ and of a degeneration of a flat family of smooth and connected curves of degree $c(s+2, t, k)$ and genus $g(s+2, t, k)$. As in the first part of step (a), restricting if necessary $\Lambda$, by semicontinuity we get $h^{i}\left(\mathcal{I}_{W(\lambda) \cup A}(s+2)\right)=0$, $i=0,1$.

(a2) To prove $M(s+2, t, k)$ we need to prove that there is a set like $A$ (call it $A^{\prime}$ ) satisfying both $h^{i}\left(\mathcal{I}_{W(\lambda) \cup A^{\prime}}(s+2)\right)=0, i=0,1$, and condition (b) of $M(s+2, t, k)$. First of all, instead of $P_{i}, 0 \leq i \leq 2$, we take a family $\left\{P_{i}(\lambda)\right\}_{\lambda \in \Lambda}$ of points of $Y$ with $P_{i}(o)=P_{i}$ and $P_{i}(\lambda) \in Y \backslash Y \cap Q$ for all $\lambda \in \Lambda \backslash\{o\}$. Assume for the moment $f=2$. We modify the definition of $D_{i}(\lambda)$, because we impose that $P_{i}(\lambda) \in D_{i}(\lambda)$ (instead of $P_{i} \in D_{i}$ ), but we also impose that $D_{1}(\lambda) \cap R_{1} \neq \emptyset$ and $D_{2}(\lambda) \cap R_{2} \neq \emptyset$ (this is possible by Remark 6 ). Then we construct $L_{i}(\lambda)$ as above. With this new definition $R_{1}$ and $R_{2}$ are secant lines of $W(\lambda) \backslash\left(C_{t, k} \cup Y\right), Y \subset W(\lambda), \pi_{1}\left(A_{2}\right) \subseteq \pi_{1}\left(A_{1}\right)$ and $\pi_{1}\left(A_{f}\right) \subseteq \pi_{1}(Q \cap(Y \backslash J(\lambda) \cap Y))$; call $m_{1}, \ldots, m_{x}, x=\sharp\left(A_{f}\right)$, the points of $Y \cap Q$ whose image is $\pi_{1}\left(A_{f}\right)$. We fix $\lambda \in \Lambda \backslash\{o\}$. Let $\left\{B_{\delta}\right\}_{\delta \in \Delta}$ be a smoothing of $W(\lambda)$ with $\Delta$ an affine and connected smooth curve, $a \in \Delta$, and $B_{a}=W(\lambda)$. Set $A(a):=A$. Since $Y$ is transversal to $Q$, up to a finite covering of $\Delta$ we may find $x+2$ sections $s_{1}, \ldots, s_{x}, z_{1}, z_{2}$ of the total space of $\left\{B_{\delta}\right\}_{\delta \in \Delta}$ with $s_{i}(a)=m_{i}$, $z_{1}(a)=R_{1} \cap D_{1}(\lambda), z_{2}(a)=R_{2} \cap D_{2}(\lambda), s_{i}(\delta) \in B_{\delta} \cap Q, z_{1}(\delta) \in B_{\delta} \cap Q$ and $z_{2}(\delta) \in B_{\delta} \cap Q$ for all $\Delta$. Let $R_{h}(\delta), h=1,2$, be the only element of $\left|\mathcal{O}_{Q}(0,1)\right|$ containing $z_{h}(\delta)$. For each $\delta \in \Delta \backslash\{a\}$ and $i \in\{1, \ldots, x\}$ let $M_{i}(\delta) \in\left|\mathcal{O}_{Q}(1,0)\right|$ be the only line of this ruling of $Q$ containing $s_{i}(\delta)$. Set $A_{1}(\delta):=\cup_{i=1}^{x}\left(R_{1}(\delta) \cap M_{i}(\delta)\right)$ and $A_{2}(\delta):=\cup_{i=1}^{d(s+2, t, k)-x}\left(R_{2}(\delta) \cap M_{i}(\delta)\right)$. Set $X_{\delta}:=C_{t, k} \cup B_{\delta}$. By construction ( $\left.X_{\delta}, Q, R_{1}, R_{2}, A_{1}(\delta), A_{2}(\delta)\right)$ satisfies condition (b) of $M(s+2, t, k)$, exchanging the two rulings of $Q$. By semicontinuity we have $h^{i}\left(\mathcal{I}_{B_{\delta} \cup A(\delta)}(s+2)\right)=0, i=0,1$, for a general $\delta \in \Delta$.

Now assume $f=1$. In this case we only impose that $D_{i}(\lambda)$ meets $R_{1}$; we have $\pi_{1}\left(A_{1}\right) \subset \pi_{1}(Q \cap(Y \backslash J(\lambda) \cap Y))$ and $x=\sharp\left(A_{1}\right)=b(s+2, t, k)$.

(b) Assume $e=1$ and $d(s, t, k)>0$, i.e. assume $0<d(s, t, k) \leq c(s+2, t, k)-$ $c(s, t, k)-3$. We set $S_{2}:=0$ and ignore $D_{2}$. We fix $o \in S_{1}$. Take a line $D_{0} \neq D_{1}$ meeting $Y \cap Q$ and $c(s+2, t, k)-c(s, t, k)-2$ distinct lines $L_{i} \in\left|\mathcal{O}_{Q}(0,1)\right|$, with $L_{i} \cap\left(C_{t, k} \cap Q\right)=\emptyset$ for all $i, L_{i} \cap(Y \cap Q) \neq \emptyset$ if and only if $1 \leq i \leq d(s, t, k)-1$ and $S_{1} \backslash\{o\}=D_{1} \cap\left(L_{1} \cup \cdots \cup L_{d(s, t, k)-1}\right)$. Set $J:=\left(D_{0} \cup D_{1}\right) \cup\left(\bigcup_{i=1}^{c(s+2, t, k)+c(s, t, k)-2} L_{i}\right)$ 
and $\chi:=\cup_{o \in S_{1}} \chi(o)$. Note that $\chi(X \cup J \cup \chi)-\chi(X)=c(s, t, k)-c(s+2, t, k)+3$. To modify step (a2) we impose that $D_{1}(\lambda) \cap R_{1} \neq \emptyset$ and $D_{0}(\lambda) \cap R_{2} \neq \emptyset$.

(c) Assume $d(s, t, k)=0$. Hence $S_{1}=S_{2}=\emptyset$. Take a line $D_{0} \in\left|\mathcal{O}_{Q}(1,0)\right|$ different from $D_{1}, D_{2}$ and with $D_{0} \cap Y \cap Q \neq \emptyset$. Take $c(s+2, t, k)-c(s, t, k)-1$ lines $L_{i} \in\left|\mathcal{O}_{Q}(0,1)\right|, 1 \leq i \leq c(s+2, t, k)-c(s, t, k)-1$, such that $L_{i} \cap\left(C_{t, k} \cap Q\right)=\emptyset$ for all $i$ and $L_{i} \cap(Y \cap Q) \neq \emptyset$ if and only if $1 \leq i \leq c(s+2, t, k)-c(s, t, k)-3$. Set $J:=D_{0} \cup\left(\bigcup_{i=1}^{c(s+2, t, k)-c(m, t, k)-1} L_{i}\right), Y^{\prime}:=Y \cup J$ and $W:=X \cup J$. Note that $\chi(W)-\chi(X)=c(s, t, k)-c(s+2, t, k)+3$. The union $Y^{\prime}$ is a connected nodal curve, which is a flat degeneration of a family of smooth curves of degree $c(s+2, t, k)$ and genus $g(s+2, t, k)$ not intersecting $C_{t, k}$. As in step (a) we get $h^{1}\left(\mathcal{I}_{W}(s+2)\right)=0$ and $h^{0}\left(\mathcal{I}_{W}(s+2)\right)=d(s+2, t, k)$. If $d(s+2, t, k)=0$, then we are done, because $A=\emptyset$ and so condition (b) of $M(s+2, t, k)$ is trivially true. Now assume $d(s+2, t, k)>0$.

First assume $f=2$. As in step (a) we prove $M(s+2, t, k)$ interchanging the rulings of $Q$ and set $x:=c(s+4, t, k)-c(s+2, t, k)-3$. We fix general lines $R_{1}, R_{2} \in$ $\left|\mathcal{O}_{Q}(0,1)\right|$ and take $A_{i} \subset R_{i}$ such that $\pi_{1}\left(A_{2}\right) \subseteq \pi_{1}\left(A_{1}\right) \cap \pi_{1}(Q \cap(Y \backslash J \cap Y))$. Set $A:=A_{1} \cup A_{2}$. For a general $X$ we have $h^{i}\left(\mathcal{I}_{W \cup A}(s+2)\right)=0, i=0,1$. Set $q:=D_{0} \cap Y$. By Remark 6 there is a family $\left\{D_{0}(\lambda)\right\}_{\lambda \in \Lambda}$ of lines of $\mathbb{P}^{3}$ and $o \in \Lambda$ with $D_{0}(o)=D_{0}, \sharp\left(D_{0}(\lambda) \cap Y\right)=1$ for all $\lambda, D_{0}(\lambda) \cap Y \notin Q$ if $\lambda \neq 0$, $D_{0}(\lambda) \cap R_{1} \neq \emptyset$ and $D_{0}(\lambda) \cap R_{2} \neq \emptyset$. Up to a finite covering of $\Lambda$ we may also find families $\left\{L_{i}(\lambda)\right\}_{\lambda \in \Lambda}, 1 \leq i \leq c(s+2, t, k)-c(s, t, k)-1$. Set $J(\lambda)=D_{0}(\lambda):=$ $D_{0} \cup\left(\bigcup_{i=1}^{c(s+2, t, k)-c(s, t, k)-1} L_{i}(\lambda)\right)$. We do the smoothing of $Y \cup J(\lambda)$ as in step (a2).

Finally, if $f=1$ we only need $D_{0}(\lambda) \cap R_{1} \neq \emptyset$ for all $\lambda$.

\section{With a constant genus $g$}

We fix an integer $t \geq 27$ and take $k \in\{t-1, t\}$. We fix an integer $g \geq g_{t, k}+g(t+$ $k+5, t, k)$. Let $y$ be the maximal integer $\geq t+k+5$ such that $y \equiv t+k-1(\bmod 2)$ and $g_{t, k}+g(y, t, k) \leq g$ (y exists, because $\left.\lim _{u \rightarrow+\infty} g(t+k+1+2 u, t, k)=+\infty\right)$. By the definition of $y$ we have $y \geq t+k+5$ and $y \equiv t+k-1(\bmod 2)$. For all integers $x \geq y+2$ with $x \equiv y(\bmod 2)$ define the integers $a(x, t, k, y)$ and $b(x, t, k, y)$ by the relation

(10) $x d_{t, k}+3-g+x a(x, t, k, y)+b(x, t, k, y)=\left(\begin{array}{c}x+3 \\ 3\end{array}\right), 0 \leq b(x, t, k, y) \leq x-1$

If $x \geq y+4$, by taking the difference between equation (10) and the same equation for the integer $x^{\prime}:=x-2$ we get

$$
\begin{array}{r}
2 d_{t, k}+2 a(x, t, k, y)+(x+2)(a(x+2, t, k, y)-a(x, t, k, y)) \\
+b(x+2, t, k, y)-b(x, t, k, y)=(x+3)^{2}
\end{array}
$$

Lemma 9. For each $x \geq y+2$ with $x \equiv y(\bmod 2)$ we have $2(a(x+2, t, k, y)-$ $a(x, t, k, y)) \geq x+5$.

Proof. Assume by contradiction $2(a(x+2, t, k, y)-a(x, t, k, y)) \leq x+4$. Recall that for all $u \geq v>0$ we have

$$
(u+v-1) d_{u, v}+2-g_{u, v}=\left(\begin{array}{c}
u+v+2 \\
3
\end{array}\right)
$$

Claim 1: We have $g_{\lceil(y+3) / 2\rceil,\lfloor(y+3) / 2\rfloor}>g$. 
Proof of Claim 1: By the definition of $y$ we have $g(y+2, t, k)+g_{t, k}>g$. Thus to prove Claim 1 it is sufficient to use that $g(y+2, t, k)+g_{t, k} \leq g_{\lceil(y+3) / 2\rceil,\lfloor(y+3) / 2\rfloor}$ (Lemma 3).

First assume $x$ odd, i.e. $k=t$. Since $g_{(x+1) / 2,(x+1) / 2} \geq g_{(y+3) / 2,(y+3) / 2}>g$ by Claim 1, (12) and (10) give $d_{(x+1) / 2,(x+1) / 2} \geq d_{t, k}+a(x, t, k, y)$. Since $b(x+$ $2, t, k, y) \leq x+1$ and $b(x, t, k, y) \geq 0$ (11) gives

$$
(x+1)(x+3) / 2+(x+2)(x+4) / 2+x+1 \geq(x+3)^{2},
$$

which is false. Now assume $x$ even, i.e. $k=t-1$. Since $g_{(x+2) / 2, x / 2} \geq g_{(y+4) / 2,(y+2) / 2}>$ $g$ by Claim 1, (12) and (10) gives $d_{(x+2) / 2, x / 2} \geq d_{t, k}+a(x, t, k, y)$. Since $b(x+$ $2, t, k, y) \leq x+1$ and $b(x, t, k, y) \geq 0$ (11) gives

$$
(x+2)^{2} / 2+(x+2)(x+4) / 2+x+1 \geq(x+3)^{2} \text {, }
$$

which is false.

Lemma 10. We have $2(a(y+2, t, k, y)-c(y, t, k)) \geq y+5$.

Proof. Define the integers $w, z$ by the relations

$$
(y+2)\left(w+d_{t, k}\right)+3-g_{t, k}-g(y, t, k)+z=\left(\begin{array}{c}
y+5 \\
3
\end{array}\right), 0 \leq z \leq y+1
$$

Since $g \geq g_{t, k}+g(y, t, k)$, we have $w \leq a(y+2, t, k)$. Hence it is sufficient to prove that $2(w-c(y, t, k)) \geq y+5$. Taking the difference between (13) and the case $s=y$ of (8) we get

$$
2 d_{t, k}+2 c(y, t, k)+(y+2)(w-c(y, t, k))+z-d(y, t, k)=(y+3)^{2}
$$

Then we continue as in the proof of Lemma 9 with $y+2$ instead of $x+2$.

The next lemma follows at once by induction on $x$, the inequality $2 c(y, t, k) \geq$ $y+6$ and Lemmas 9 and 10 .

Lemma 11. We have $2 a(x, t, k, y) \geq x+6$ for all integers $x \geq y+2$ with $x \equiv y$ $(\bmod 2)$.

Lemma 12. For each $x \geq y+2$ with $x \equiv y(\bmod 2)$ we have $a(x, t, k, y) \geq$ $g-g_{t, k}+3$.

Proof. First assume $x=y+2$. By the definition of the integer $y$ we have $g_{t, k}+$ $g(y, t, k) \leq g \leq \tau:=g_{t, k}+g(y+2, t, k)-1$. The integers $a(y+2, t, k, y)$ and $b(y+2, t, k, y)$ depend on the choice of $g$ and (only for this proof) we call them $a(y+2, t, k, y)_{g}$ and $a(y+2, t, k, y)_{g}$. Fix integers $q, q^{\prime}$ such that $g_{t, k}+g(y, t, k) \leq$ $q \leq q^{\prime} \leq \tau$. From (10) or (11) for $q$ and $q^{\prime}$ we get

$$
\begin{aligned}
& (y+2)\left(a(y+2, t, k, y)_{q^{\prime}}-a(y+2, t, k, y)_{q}\right)= \\
& b(y+2, t, k, y)_{q}-b(y+2, t, k, y)_{q^{\prime}}+q^{\prime}-q
\end{aligned}
$$

Since $0 \leq b(y+2, t, k, y)_{q} \leq y+1$ and $0 \leq b(y+2, t, k, y)_{q^{\prime}} \leq y+1$, (14) implies $a(y+2, t, k, y)_{q} \leq a(y+2, t, k, y)_{q^{\prime}} \leq a(y+2, t, k, y)_{q}+q^{\prime}-q$. Thus to prove the lemma for $x=y+2$ it is sufficient to prove it for the genus $\tau$. We have, by (8) and (10),

$$
\begin{aligned}
& (y+2)\left(d_{t, k}+c(y+2, t, k)\right)+3-g_{t, k}-g(y+2, t, k)+d(y+2, t, k)= \\
& (y+2)\left(d_{t, k}+a(y+2, t, k, y)_{\tau}\right)+b(y+2, t, k, y)_{\tau}+3-\tau
\end{aligned}
$$


Hence

(15) $(y+2)\left(c(y+2, t, k)-a(y+2, t, k, y)_{\tau}\right)=-d(y+2, t, k)+b(y+2, t, k, y)_{\tau}+1$

From (15) we get $a(y+2, t, k, y)_{\tau} \geq c(y+2, t, k)-1$. Since $c_{y+2, t, k} \geq g(y+2, t, k)+$ $3=\tau-g_{t, k}+4$, we get $a(y+2, t, k, y)_{\tau} \geq \tau-g_{t, k}+3$.

Now assume $x \geq y+4$. By Lemma 9 we have $a(x, t, k, y) \geq a(y+2, t, k, y)$.

By Lemma 12 there is a non-special curve of degree $a(x, t, k, y)$ and genus $g-g_{t, k}$. We need this observation in the next statement.

Assertion $N(x, t, k, y), x \geq y, x \equiv y(\bmod 2):$ Set $e=1$ if $0 \leq b(x, t, k, y) \leq$ $a(x+2, t, k, y)-a(x, t, k, y)-1$ and $e=2$ if $b(x, t, k, y) \geq a(x+2, t, k, y)-a(x, t, k, y)$. There is a 6 -tuple $\left(X, Q, D_{1}, D_{2}, S_{1}, S_{2}\right)$ such that

(a) $Q$ is a smooth quadric surface, $X=C_{t, k} \sqcup Y, Y$ is a smooth non-special curve of degree $a(x, t, k, y)$ and genus $g-g_{t, k}$ and $Q$ intersects transversally $X$, with no line of $Q$ containing $\geq 2$ points of $X \cap Q$;

(b) $D_{1}, D_{2}$ are different elements of $\left|\mathcal{O}_{Q}(1,0)\right|$, each of them containing one point of $Y \cap Q, S_{i} \subset D_{i} \backslash D_{i} \cap Y, 1 \leq i \leq 2$, and $\sharp\left(S_{1}\right)+\sharp\left(S_{2}\right)=b(x, t, k, y)$; $\pi_{2}\left(S_{2}\right) \subseteq \pi_{2}\left(S_{1}\right)$ and $\pi_{2}\left(S_{e}\right) \subset \pi_{2}\left(Y \cap\left(Q \backslash\left(D_{1} \cup D_{2}\right)\right)\right) ; S_{2}=\emptyset$ if $e=1$, $\sharp\left(S_{2}\right)=b(x+2, t, k, y)-a(x+2, t, k, y)+a(x, t, k, y)+2$ if $e=2$;

(c) $h^{i}\left(\mathcal{I}_{X \cup S_{1} \cup S_{2}}(x)\right)=0, i=0,1$.

Lemma 13. If $N(x, t, k, y)$ is true, then $N(x+2, t, k, y)$ is true.

Proof. We outline the modifications of the proof of Lemma 8 needed to get Lemma 13 Let $e \in\{1,2\}$ (resp. $f \in\{1,2\}$ ) be the integer arising in $N(x, t, k, y)$ (resp. $N(x+2, t, k, y))$. Take $\left(X, Q, D_{1}, D_{2}, S_{1}, S_{2}\right)$ satisfying $N(x, t, k, y)$. Set $w:=$ $a(x+2, t, k, y)-a(x, t, k, y)$.

(a) Assume $e=2$. Set $z:=b(x+2, t, k, y)+2-w$. Since $b(x+2, t, k, y) \leq x+1$, Lemma 9 gives $z \leq w-2$. Let $L_{i} \in\left|\mathcal{O}_{Q}(0,1)\right|, 1 \leq i \leq w-2$, be the lines such that $S_{1}=D_{1} \cap\left(L_{1} \cup \cdots \cup L_{w-2}\right)$ and $S_{2}=D_{2} \cap\left(L_{1} \cup \cdots \cup L_{z}\right)$. Set $J:=D_{1} \cup D_{2} \cup\left(\bigcup_{i=1}^{w-2} L_{i}\right)$

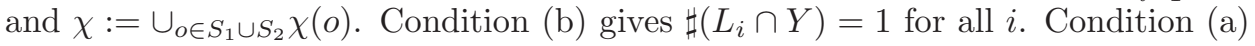
gives $C_{t, k} \cap J=\emptyset$. Hence $W:=X \cup J \cup \chi$ is a smoothable curve of degree $a(x+2, t, k, y)$ with $h^{1}\left(\mathcal{O}_{W}\right)=g$.

(b) Assume $e=1$, i.e. assume $d(x+2, t, k, y) \leq w-1$. Let $L_{i} \in\left|\mathcal{O}_{Q}(0,1)\right|$, $1 \leq i \leq b(x, t, k, y)$, be the lines such that $S_{1}=D_{1} \cap\left(L_{1} \cup \cdots \cup L_{b(x, t, k, y)}\right)$. Take general lines $L_{j} \in\left|\mathcal{O}_{Q}(0,1)\right|, b(x, t, k, y)<j \leq w-1$. Set $J:=D_{1} \cup\left(\bigcup_{i=1}^{w-1} L_{i}\right)$ and $\chi:=\cup_{o \in S_{1}} \chi(o)$. Condition (a) gives $C_{t, k} \cap J=\emptyset$. Hence $W:=X \cup J \cup \chi$ is a smoothable curve of degree $a(x+2, t, k, y)$ with $h^{1}\left(\mathcal{O}_{W}\right)=g$.

Lemma 14. $N(y+2, t, k, y)$ is true.

Proof. Use the proof of Lemma 8 and Lemma 13 starting with $\left(X, Q, D_{1}, D_{2}, S_{1}, S_{2}\right)$ satisfying $M(y, t, k)$ and quoting Lemma 10 instead of Lemma 9

\section{Proving Conjecture 1}

In order to prove Theorem 1 and Corollary 1 first of all we notice that from the previous section we could deduce with a small effort the following two facts, but that (as explained at the end of the introduction) they would not prove Theorem 1 and Corollary 1 . 
For each integer $d$ such that $g-3 \leq d \leq d(m, g)_{\max }$ there exists a smooth and connected curve $X_{1} \subset \mathbb{P}^{3}$ such that $\operatorname{deg}\left(X_{1}\right)=d, g(X)=g, h^{1}\left(\mathcal{O}_{X_{1}}(m-2)\right)=0$, $h^{1}\left(\mathcal{I}_{X_{1}}(m)\right)=0$ and $h^{1}\left(N_{X_{1}}(-1)\right)=0$.

For each integer $d \geq d(m, g)_{\text {min }}$ there exists a smooth and connected curve $X_{2} \subset \mathbb{P}^{3}$ such that $\operatorname{deg}\left(\bar{X}_{2}\right)=d, g(X)=g, h^{1}\left(\mathcal{O}_{X_{2}}(m-2)\right)=0, h^{0}\left(\mathcal{I}_{X_{2}}(m-1)\right)=0$ and $h^{1}\left(N_{X_{2}}(-1)\right)=0$.

Now fix an integer $d$ such that $d(m, g)_{\min } \leq d \leq d(m, g)_{\max }$. To prove Theorem 1 for the pair $(d, g)$ it is sufficient to prove that we may find $X_{1}, X_{2}$ as above and with the additional condition that $X_{1}$ and $X_{2}$ are in the same irreducible component, $\Gamma$, of $\operatorname{Hilb}\left(\mathbb{P}^{3}\right)$. If we prove this statement, then by the semicontinuity theorem for cohomology $\left(\left[15\right.\right.$, III.8.8]) we get $h^{1}\left(\mathcal{I}_{X}(m)\right)=0$ and $h^{0}\left(\mathcal{I}_{X}(m-1)\right)=0$, hence we would conclude the proof for the pair $(d, g)$. To get $X_{1}$ and $X_{2}$ in the same irreducible component of $\operatorname{Hilb}\left(\mathbb{P}^{3}\right)$ we need to rewrite the proofs of the previous section with a few improvements. But first we need to distinguish between the case in which $d$ is very near to $d(m, g)_{\min }$ and the case in which $d$ is very near to $d(m, g)_{\max }$. In the first case (say $\left.d(m, g)_{\min } \leq d \leq d^{\prime}\right)$ we will modify the proof of the existence of $X_{2}$ with $h^{0}\left(\mathcal{I}_{X_{2}}(m-1)\right)=0$ to get (for the same curve $X_{2}$ ) also $h^{1}\left(\mathcal{I}_{X_{2}}(m)\right)=0$. If $d$ is very near to $d(m, g)_{\max }$ (say $d^{\prime \prime} \leq d \leq d(m, g)_{\max }$ ) we will modify the proof of the existence of the curve $X_{1}$ to get a curve $X_{1}$ with $h^{1}\left(\mathcal{I}_{X_{1}}(m)\right)=0$ and $h^{0}\left(\mathcal{I}_{X_{1}}(m-1)\right)=0$. We use that $N(x, t, k, y)$ are true for $x=m-5, m-4, m-3, m-2$ (Lemma 15).

Set $\varepsilon:=0$ if $m$ is odd and $\varepsilon:=1$ if $m$ is even.

5.0.1. Near $d(m, g)_{\min }$. In this range the most difficult part is the proof of the existence of $X_{2}$. It is the construction of $X_{2}$ which says in which $W\left(t^{\prime}, k^{\prime}, d^{\prime}, b^{\prime}\right)$ we will try to find $X_{1}$. Recall that to get a curve $X_{2}$ with $h^{0}\left(\mathcal{I}_{X_{2}}(m-1)\right)=0$ we started with a curve $C_{t, t-\varepsilon}$ with $h^{i}\left(\mathcal{I}_{C_{t, t-\varepsilon}}(2 t-1-\varepsilon)\right)=0$, where $t$ is the maximal integer $t>0$ such that such that $g_{t, t-\varepsilon}+g(2 t+5-\varepsilon, t, t-\varepsilon) \leq g$. Set $k:=t-\varepsilon$. Recall that an element $W$ of $U\left(t, k, a_{d}, b\right)$ has degree $d$ and $h^{1}\left(\mathcal{O}_{W}\right)=g$ if and only if $b=g-g_{t, k}$ and $a_{d}=d-d_{t, k}$. The component $W\left(t^{\prime}, k^{\prime}, d^{\prime}, b^{\prime}\right)$ is the component $W\left(t, k, a_{d}, b\right)$, where $b=g-g_{t, k}$ and $a_{d}=d-d_{t, k}$. The curve $T$ satisfying $N(m-1, t, k, y)$ has $h^{1}\left(\mathcal{O}_{T}\right)=g, 3$ connected components, $h^{0}\left(\mathcal{I}_{T}(m-1)\right)=b(m-1, t, k, y)$ and $h^{1}\left(\mathcal{I}_{T}(m-1)\right)=0$, hence $d>a(m-1, t, k, y)+d_{t, k}$. The minimum integer $d(m, g)_{\min }$ is $a(m-1, t, k, y)+d_{t, k}+1$, unless $b(m-1, t, k, y) \in\{m-2, m-1\}$ (in the latter case we have $\left.d(m, g)_{\min }=a(m-1, t, k, y)+d_{t, k}+2\right)$.

(a) We make the construction of Section 4 for the integer $m^{\prime}:=m-1 \equiv t+k-1$ ( $\bmod 2)$ and the integer $g$ (note that the numerology for $g$ in Theorem 1 is such that we may do the construction of Section 4 for $m^{\prime}:=m-1$ and the integer $g)$. We get an integer $y \leq m^{\prime}-4=m-5$ with $y \equiv t+k-1 \equiv 0(\bmod 2)$. Then for all integers $x \geq y+2$ with $x \equiv y(\bmod 2)$ we proved $N(x, t, k, y)$. Hence $N(m-5, t, k, y)$ and $N(m-3, t, k, y)$ are true (Lemma 15). Since $d \geq d(m, g)_{\min }$, we have $d>a(m-1, t, k, y)+d_{t, k}$, hence we want to add in a smooth quadric $Q$ a certain union of $d-a(m-3, t, k, y)-d_{t, k}$ lines. We write $C_{t} \cup C_{k}^{\prime}$ for a general (but fixed in this construction) $C_{t, k}$, because we need to distinguish the two connected components of $C_{t, k}$, even when $k=t$.

(a1) Assume $d=d(m, g)_{\min }=a(m-1, t, k, y)+d_{t, k}+1$. Set $z:=d-$ $a(m-3, t, k, y)-d_{t, k}=1+a(m-1, t, k, y)-a(m-3, t, k, y)$. We need to modify $N(m-3, t, k, y)$ in the following way. 
Assertion $N^{\prime}(m-3, t, k, y), m-3 \equiv y(\bmod 2):$ Set $e=1$ if $b(m-3, t, k, y) \leq$ $z-3$ and $e=2$ if $b(m-3, t, k, y) \geq z-2$. There is a 6 -tuple $\left(X, Q, D_{1}, D_{2}, S_{1}, S_{2}\right)$ such that

(a) $Q$ is a smooth quadric surface, $X=C_{t} \sqcup C_{k}^{\prime} \sqcup Y, Y$ is a smooth curve of degree $a(m-3, t, k, y)$ and genus $g-g_{t, k}$ and $Q$ intersects transversally $X$, with no line of $Q$ containing $\geq 2$ points of $X \cap Q$;

(b) $D_{1}, D_{2}$ are different elements of $\left|\mathcal{O}_{Q}(1,0)\right|, D_{1} \cap C_{t} \neq \emptyset, D_{2} \cap C_{k} \neq \emptyset$, $S_{i} \subset D_{i} \backslash D_{i} \cap\left(C_{t} \cup C_{k}^{\prime}\right), 1 \leq i \leq 2$, and $\sharp\left(S_{1}\right)+\sharp\left(S_{2}\right)=b(m-3, t, k, y)$; $\pi_{2}\left(S_{2}\right) \subseteq \pi_{2}\left(S_{1}\right), \pi_{2}\left(S_{e}\right) \subset \pi_{2}\left(Y \cap\left(Q \backslash\left(D_{1} \cup D_{2}\right)\right)\right) ; S_{2}=\emptyset$ if $e=1$, $\sharp\left(S_{2}\right)=b(m-3, t, k, y)-z+3$ if $e=2$;

(c) $h^{i}\left(\mathcal{I}_{X \cup S_{1} \cup S_{2}}(m-3)\right)=0, i=0,1$.

As in the proof of Lemma 8 and Lemma 13 we get $\left(X, Q, D_{1}, D_{2}, S_{1}, S_{2}\right), X=$ $C_{t} \sqcup C_{k}^{\prime} \sqcup Y$ satisfying $N^{\prime}(m-3, t, k, y)$; in the proof of Lemma 8 we take $R_{1}$ containing a point of $C_{t} \cap Q$ instead of a point of $Y \cap Q$ and $R_{2}$ containing a point of $C_{k}^{\prime} \cap Q$ instead of a point of $Y \cap Q$.

(a1.1) Assume $b(m-3, t, k, y)=0$. Take $D_{0} \in\left|\mathcal{O}_{Q}(1,0)\right|$ containing one point of $Y \cap Q, L_{1} \in\left|\mathcal{O}_{Q}(0,1)\right|$ containing a point of $C_{t}, L_{2} \in\left|\mathcal{O}_{Q}(0,1)\right|$ containing a point of $C_{k}^{\prime}$ and general $L_{i} \in\left|\mathcal{O}_{Q}(0,1)\right|, 3 \leq i \leq z-1$. Set $J:=D_{0} \cup\left(\bigcup_{i=1}^{z-1} L_{i}\right)$. Since $X \cap(Q \backslash J)$ is a general subset of $Q$ with cardinality $2 d_{t, k}+2 a(m-3, t, k, y)-3$, we have $h^{0}\left(Q, \mathcal{I}_{Q \cap(X \cup J)}(m-1)\right)=h^{0}\left(Q, \mathcal{I}_{X \cap(Q \backslash J)}(m-2, m-z)\right)=0$ (use (10) for $x=$ $m-3$, that $z=1+a(m-1, t, k, y)-a(m-3, t, k, y)$ and that $b(m-1, t, k, y) \leq m-2)$. Since $\operatorname{Res}_{Q}(X \cup Y)=X$ and $h^{0}\left(\mathcal{I}_{X}(m-3)\right)=0$, we have $h^{0}\left(\mathcal{I}_{X \cup J}(m-1)\right)=0$. The union $X \cup J$ is a nodal and connected smoothable curve of degree $d$ and arithmetic genus $g$ and $Y \cup J$ is a connected smoothable curve of degree $d-d_{t, k}$ and arithmetic genus $g-g_{t, k}-2 \geq 26$. We may smooth $Y \cup J$ in a family of curves, all of them containing the two points $\left(C_{t} \cup C_{k}^{\prime}\right) \cap J$. Call $E$ a general element of this smoothing. Since Aut $\left(\mathbb{P}^{3}\right)$ is 2 -transitive, we may see $E$ as a general non-special space curve of its degree and its genus $\geq 26$. By construction and Lemma2 we have $C_{t} \cup C_{k}^{\prime} \cup E \in U\left(t, k, a_{d}, b\right)$ and $h^{1}\left(N_{C_{t} \cup C_{k}^{\prime} \cup E}(-1)\right)=0$. By semicontinuity there is a smooth $X_{2} \in W\left(t, k, a_{d}, b\right)$ with $h^{0}\left(\mathcal{I}_{X_{2}}(m-1)\right)=0$ and $h^{1}\left(N_{X_{2}}(-1)\right)=0$.

(a1.2) Assume $0<b(m-3, t, k, y) \leq z-3$. Hence $S_{2}=\emptyset$. We take $D_{1}$ and call $L_{i} \in\left|\mathcal{O}_{Q}(0,1)\right|, 1 \leq i \leq b(m-3, t, k, y)$, the elements of $\left|\mathcal{O}_{Q}(0,1)\right|$ such that $S_{1}=D_{1} \cap\left(L_{1} \cup \cdots \cup L_{b(m-3, t, k, y)}\right)$; note that each line $L_{i}$ contains a point of $Y \cap Q$. Take any $L_{b(m-3, t, k, y)+1} \in\left|\mathcal{O}_{Q}(0,1)\right|$ with $C_{k}^{\prime} \cap L_{b(m-3, t, k, y)+1} \neq \emptyset$, any $L_{b(m-3, t, k, y)+2} \in\left|\mathcal{O}_{Q}(0,1)\right|$ with $Y \cap L_{b(m-3, t, k, y)+2} \neq \emptyset, L_{b(m-3, t, k, y)+2} \neq L_{i}$ for $i \leq b(m-3, t, k, y)$ and (if $b(m-3, t, k, y)<z-3)$ take general $L_{j} \in\left|\mathcal{O}_{Q}(0,1)\right|$, $b(m-3, t, k, y)+3 \leq j \leq z-1$. Set $J:=D_{1} \cup\left(\bigcup_{i=1}^{z-1} L_{i}\right), \chi:=\cup_{o \in S_{1}} \chi(o)$ and $W:=X \cup J \cup \chi$. We have $\operatorname{Res}_{Q}(W)=X \cup S_{1}$ and thus $h^{0}\left(\mathcal{I}_{\operatorname{Res}_{Q}(W)}(m-3)\right)=0$. Since $W \cap Q$ is the union of $J$ and $2 d_{t, k}+2 a(m-3, t, k, y)-b(m-3, t, k, y)-3$ general points of $Q$ and $b(m-1, t, k, y) \leq m-1$, (11) gives $h^{0}\left(Q, \mathcal{I}_{W \cap Q}(m-1)\right)=$ $h^{0}\left(Q, \mathcal{I}_{X \cap(Q \backslash J)}(m-2, m-z)\right)=0$. Thus $h^{0}\left(\mathcal{I}_{W}(m-1)\right)=0$. We first deform $W$ to the union $F$ of $C_{t} \cup C_{k}^{\prime} \cup D_{1} \cup Y \cup\left(\bigcup_{i=b(m-3, t, k, y)+1}^{z-1} L_{i}\right)$ and $b(m-3, t, k, y)$ disjoint lines $M_{1}, \ldots, M_{b(m-3, t, k, y)}$, each of them containing one point of $Y$. The union $F$ is a nodal and connected curve. Write $F=C_{t} \cup C_{k}^{\prime} \cup G$. We have $\sharp\left(G \cap C_{t}\right)=\sharp\left(G \cap C_{k}^{\prime}\right)=1$. Let $G^{\prime}$ be a general smoothing of $G$ fixing the 2 points of $\left(C_{t} \cup C_{k}^{\prime}\right) \cap G$. $C_{t} \cup C_{k}^{\prime} \cup G^{\prime} \in U\left(t, k, a_{d}, b\right)$. By Lemma2 2 and semicontinuity there is a smooth $X_{2} \in W\left(t, k, a_{d}, b\right)$ with $h^{0}\left(\mathcal{I}_{X_{2}}(m-1)\right)=0$ and $h^{1}\left(N_{X_{2}}(-1)\right)=0$. 
(a1.3) Assume $b(m-3, t, k, y) \geq z-2$. Since $z=a(m-1, t, k, y)-a(m-$ $3, t, k, y)+1$ and $b(m-3, t, k)) \leq m-4$, Lemma 9 gives $2(z-3) \geq b(m-3, t, k, y)$. Let $L_{i} \in\left|\mathcal{O}_{Q}(0,1)\right|, 1 \leq i \leq z-3$, be the lines such that $S_{1}=D_{1} \cap\left(\bigcup_{i=1}^{z-3} L_{i}\right)$ and $S_{2}:=D_{2} \cap\left(\bigcup_{i=1}^{w} L_{i}\right)$. Take $L_{z-2} \in\left|\mathcal{O}_{Q}(0,1)\right|$ containing one point of $Y \cap Q$ and different from the other lines $L_{i}, i \leq z-3$. Set $J:=D_{1} \cup D_{2} \cup\left(\cup_{i=1}^{z-2} L_{i}\right)$, $\chi:=\cup_{o \in S_{1}} \chi(o)$ and $W:=X \cup J \cup \chi$. We have $\operatorname{Res}_{Q}(W)=X \cup S_{1} \cup S_{2}$ and thus $h^{0}\left(\mathcal{I}_{\operatorname{Res}_{Q}(W)}(m-3)\right)=0$. Since $W \cap Q$ is the union of $J$ and $2 d_{t, k}+2 a(m, t, k, y)-w-$ 3 general points of $Q$ and $b(m-1, t, k, y) \leq m-1$ (11) gives $h^{0}\left(Q, \mathcal{I}_{W \cap Q}(m-1)\right)=$ $h^{0}\left(Q, \mathcal{I}_{X \cap(Q \backslash J)}(m-2, m-z)\right)=0$. Thus $h^{0}\left(\mathcal{I}_{W}(m-1)\right)=0$. We first deform $W$ to the union $F$ of $C_{t} \cup C_{k}^{\prime} \cup D_{1} \cup D_{2} \cup Y \cup\left(\cup_{i=w+1}^{z-2} L_{i}\right)$ and $w$ disjoint lines $M_{1}, \ldots, M_{w}$, each of them containing one point of $Y$. The union $F$ is a nodal and connected curve. Write $F=C_{t} \cup C_{k}^{\prime} \cup G$. We have $\sharp\left(G \cap C_{t}\right)=\sharp\left(G \cap C_{k}^{\prime}\right)=1$. Let $G^{\prime}$ be a general smoothing of $G$ fixing the 2 points of $\left(C_{t} \cup C_{k}^{\prime}\right) \cap G$. We have $C_{t} \cup C_{k}^{\prime} \cup G^{\prime} \in U\left(t, k, a_{d}, b\right)$. By Lemma 2 and semicontinuity there is a smooth $X_{2} \in W\left(t, k, a_{d}, b\right)$ with $h^{0}\left(\mathcal{I}_{X_{2}}(m-1)\right)=0$ and $h^{1}\left(N_{X_{2}}(-1)\right)=0$.

(a1.4) Assume $d(m, g)_{\min }=a(m-1, t, k, y)+d_{t, k}+2$. We are in the set-up of step (a1.3) with the integer $z^{\prime}:=a(m-1, t, k, y)-a(m-3, t, k, y)+2$ instead of the integer $z:=a(m-1, t, k, y)-a(m-3, t, k, y)+1$.

(a2) Assume $d>d(m, g)_{\min }$ and set $w:=d-d(m, g)_{\min }$. By step (a1) there is a nodal curve $E=C_{t} \cup C_{k}^{\prime} \cup F \in U\left(t, k, a_{d}-w, b\right)$ with $\sharp\left(C_{t} \cap F\right)=\sharp\left(C_{k}^{\prime} \cap F\right)=1$, $C_{t} \cap D_{k}^{\prime}=\emptyset, F$ and $h^{0}\left(\mathcal{I}_{E}(m-1)\right)=0$. Take a general union $G$ of $F$ and $w$ lines, each of them meeting $F$ at exactly one point and quasi-transversally. By construction $E^{\prime}:=C_{t} \cup C_{k}^{\prime} \cup G$ is nodal and $C_{t} \cap G=C_{t} \cap F, C_{k}^{\prime} \cap G=C_{k}^{\prime} \cap F$. Since $h^{0}\left(\mathcal{I}_{E}(m-1)\right)=0$ and $E^{\prime} \supset E$, we have $h^{0}\left(\mathcal{I}_{E^{\prime}}(m-1)\right)=0$. We may smooth $G$ keeping fixed the points $C_{t} \cap F$ and $C_{k}^{\prime} \cap F$, because Aut $\left(\mathbb{P}^{3}\right)$ is 2-transitive. Hence there is a non-special smooth curve $G^{\prime \prime}$ of degree $d-d_{t, k}$ and genus $g-g_{t, k}$ with $C_{t} \cap G^{\prime \prime}=C_{t} \cap F, C_{k}^{\prime} \cap G^{\prime \prime}=C_{k}^{\prime} \cap F$ and which is a general member of a family with $F^{\prime}$ as its special member and with $C_{t} \cup C_{k}^{\prime} \cup G^{\prime \prime}$ nodal. By semicontinuity we have $h^{0}\left(\mathcal{I}_{C_{t} \cup C_{k}^{\prime} \cup G^{\prime \prime}}(m-1)\right)=0$. We have $C_{t} \cup C_{k}^{\prime} \cup G^{\prime \prime} \in U\left(t, k, a_{d}, b\right)$.

(b) Set $\alpha:=t(t-2)$ if $k=t$ and $\alpha:=t^{2}-3 t+1$ if $k=t-1$. Fix a plane $H$, a smooth conic $D \subset H$ and general $C_{t, k}$. We have $D \cap C_{t, k}=\emptyset$ and $C_{t, k} \cap H$ is a general subset of $H$ with cardinality $d_{t, k}$. Hence $h^{0}\left(H, \mathcal{I}_{H \cap\left(C_{t, k} \cup D\right)}(t+k)\right)=$ $h^{0}\left(H, \mathcal{I}_{C_{t, k} \cap H}(t+k-1)\right)=\left(\begin{array}{c}t+k+1 \\ 2\end{array}\right)-d_{t, k}=\alpha$ and $h^{1}\left(H, \mathcal{I}_{H \cap\left(C_{t, k} \cup D\right)}(t+k)\right)=0$. Then we continue the construction from the critical value $t+k$ to the critical value $t+k+2$, then to the critical value $t+k+4$, and so on up to the critical value $m-2$; in each step, say to arrive at the critical value $x$ from a curve $A^{\prime}$ and a set $S^{\prime}$ with $h^{1}\left(\mathcal{I}_{A^{\prime} \cup S^{\prime}}(x-2)\right)=0$ and $h^{0}\left(\mathcal{I}_{A^{\prime} \cup S^{\prime}}(x-2)\right)=\alpha$ and $0 \leq \sharp\left(S^{\prime}\right) \leq x-3$ (and so $\sharp\left(S^{\prime}\right)=\left(\begin{array}{c}x+1 \\ 3\end{array}\right)-(x-2) \operatorname{deg}\left(A^{\prime}\right)-3+g-\alpha$; we have bijectivity inside $Q$ and get a curve $A^{\prime \prime}$ and a set $S^{\prime \prime}$ with $h^{1}\left(\mathcal{I}_{A^{\prime \prime} \cup S^{\prime \prime}}(x)\right)=0$ and $h^{0}\left(\mathcal{I}_{A^{\prime \prime} \cup S^{\prime \prime}}(x)\right) \leq \alpha$. In the last step we also need to connect the connected components of the curve and get an element $B \in U\left(t, k, a^{\prime}, b\right)$ for some $a^{\prime}$; we need to check that at each step the numerical conditions are satisfied. Call $\left(X, Q, D_{1}, D_{2}, S_{1}, S_{2}\right)$ the curve we get for $\mathcal{O}_{\mathbb{P}^{3}}(m-2)$ and either $e=1$ or $e=2$. Set $S:=S_{1} \cup S_{2}$ and $\alpha^{\prime}:=\sharp(S)$. We have $0 \leq \alpha^{\prime} \leq m-3$. Since $S$ is a union of connected components of $X \cup S$, the restriction map $H^{0}\left(\mathcal{O}_{X \cup S}(m-2)\right) \rightarrow H^{0}\left(\mathcal{O}_{X}(m-2)\right)$ is surjective and its kernel has dimension $\sharp(S)$. Since $h^{1}\left(\mathcal{I}_{X \cup S}(m-2)\right)=0$, we have $h^{1}\left(\mathcal{I}_{X}(m-2)\right)=0$ and $h^{0}\left(\mathcal{I}_{X}(m-2)=\alpha+\alpha^{\prime} \leq \alpha+m-3\right.$. We cover in this way the integers 
$d$ such that $\left(\begin{array}{c}m+3 \\ 3\end{array}\right)+g-1-d m \geq \alpha+m-3$. Hence we cover all $d$ such that $d(m, g)_{\max }-d \geq 1+\lfloor\alpha / m\rfloor$. If $t \leq m / 4$ we have $\alpha / m \leq m / 4$.

5.0.2. Near $d(m, g)_{\max }$. In this range the most difficult part is the existence of $X_{1}$ with $h^{1}\left(\mathcal{I}_{X_{1}}(m)\right)=0$ and it is this part which dictates the component $W\left(t^{\prime}, k^{\prime}, a^{\prime}, b^{\prime}\right)$ in which we will find both $X_{1}$ and $X_{2}$. We stress that the integers $t, k$ introduced in this subsection are not the same as in the previous one and hence also $y$ may be different.

(a) In this step we prove the existence of $X_{1}$. We start with the maximal integer $k$ such that $g_{k+1-\varepsilon, k}+g(2 k+6-\varepsilon, k+1-\varepsilon, k) \leq g$ and set $t:=k+1-\varepsilon$. We use $N(x, t, k, y)$. In particular we have $N(m-4, t, k, y)$ and $N(m-2, t, k, y)$. Set $a_{d}:=d-d_{t, k}$ and $b:=g-g_{t, k}$. In this step we prove the existence of $A \in U\left(t, k, a_{d}, b\right)$ with $h^{1}\left(\mathcal{I}_{A}(m)\right)=0$, hence by semicontinuity the existence of $X_{1} \in W\left(t, t-1, a_{d}, b\right)$ with $h^{1}\left(\mathcal{I}_{X_{1}}(m)\right)=0$. Set $z:=d-a(m-2, t, k, y)-d_{t, k}$. We write $C_{t} \cup C_{k}^{\prime}$ for a general (but fixed in this construction) $C_{t, k}$, because we need to distinguish the two connected components, even when $k=t$. Recall that we have (1).

(a1) Assume $d=d(m, g)_{\max }$. Let $T$ be any curve satisfying $N(m, t, k, y)$. We have $\operatorname{deg}(T)=d_{t, k}+a(m, t, k, y), h^{1}\left(\mathcal{O}_{T}\right)=g, h^{1}\left(\mathcal{O}_{T}(m)\right)=0, T$ has 3 connected components, $h^{1}\left(\mathcal{I}_{T}(m)\right)=0$ and $h^{0}\left(\mathcal{I}_{T}(m)\right)=b(m, t, k, y)$. By (11) we have $d=a(m, t, k, y)+d_{t, k}$ if $b(m, t, k, y) \leq m-3$ and $d=a(m, t, k, y)+d_{t, k}+1$ if $m-2 \leq b(m, t, k, y) \leq m-1$. Hence $a(m, t, k, y)-a(m-2, t, k) \leq z \leq a(m, t, k, y)-$ $a(m-2, t, k, y)+1$. Call $\eta$ the difference between the right hand side and the left hand side of (11).

Assertion $N^{\prime \prime}(m-2, t, k, y), m \equiv y(\bmod 2):$ Set $e=1$ if $b(m-2, t, k, y) \leq$ $z-3$ and $e=2$ if $b(x, t, k, y) \geq z-2$. There is a 6 -tuple $\left(X, Q, D_{1}, D_{2}, S_{1}, S_{2}\right)$ such that

(a) $Q$ is a smooth quadric surface, $X=C_{t} \sqcup C_{k}^{\prime} \sqcup Y, Y$ is a smooth curve of degree $a(m-2, t, k, y)$ and genus $g-g_{t, k}$ and $Q$ intersects transversally $X$, with no line of $Q$ containing $\geq 2$ points of $X \cap Q$;

(b) $D_{1}, D_{2}$ are different elements of $\left|\mathcal{O}_{Q}(1,0)\right|, D_{1} \cap C_{t} \neq \emptyset, D_{2} \cap C_{k}^{\prime} \neq \emptyset$, $S_{i} \subset D_{i} \backslash D_{i} \cap\left(C_{t} \cup C_{k}^{\prime}\right), 1 \leq i \leq 2$, and $\sharp\left(S_{1}\right)+\sharp\left(S_{2}\right)=b(x, t, k, y)$; $\pi_{2}\left(S_{2}\right) \subseteq \pi_{2}\left(S_{1}\right)$ and $\pi_{2}\left(S_{e}\right) \subset \pi_{2}\left(Y \cap\left(Q \backslash\left(D_{1} \cup D_{2}\right)\right)\right) ; S_{2}=\emptyset$ if $e=1$, $\sharp\left(S_{2}\right)=b(m-2, t, k, y)-z+2$ if $e=2$;

(c) $h^{i}\left(\mathcal{I}_{X \cup S_{1} \cup S_{2}}(x)\right)=0, i=0,1$.

As in the proof of Lemma 8 and Lemma 13 we get $\left(X, Q, D_{1}, D_{2}, S_{1}, S_{2}\right), X=$ $C_{t} \sqcup C_{k}^{\prime} \sqcup Y$ satisfying $N^{\prime \prime}(m-2, t, k, y)$; in the proof of Lemma 8 we take $R_{1}$ containing a point of $C_{t} \cap Q$ instead of a point of $Y \cap Q$ and $R_{2}$ containing a point of $C_{k}^{\prime} \cap Q$ instead of a point of $Y \cap Q$.

(a1.1) Assume $b(m-2, t, k, y)=0$. Take $z-1$ distinct lines $L_{i} \in\left|\mathcal{O}_{Q}(0,1)\right|$, $1 \leq i \leq z-1$, such that $L_{i} \cap C_{t}=\emptyset$ for all $i, L_{i} \cap C_{k}^{\prime} \neq \emptyset$ if and only if $i=1$ and $L_{i} \cap Y \neq \emptyset$ if and only if $i=2$. Set $J:=D_{1} \cup\left(\bigcup_{i=1}^{z-1} L_{i}\right)$. Since $X \cap(Q \backslash J)$ is a general subset of $Q$ with cardinality $2 d_{t, k}+2 a(m-3, t, k, y)-3$, we have $h^{1}\left(Q, \mathcal{I}_{Q \cap(X \cup J)}(m)\right)=h^{1}\left(Q, \mathcal{I}_{X \cap(Q \backslash J)}(m-1, m+1-z)\right)=0$ (use the generality of $X \cap(Q \backslash J)$ and the difference between (11) and the case $x:=m-2$ of (10), which gives an upper bound for $\sharp(X \cap(Q \backslash J))$; we get an equality if and only if $\eta=0$, i.e. $b(m, t, k, y)=m-2$ and $\left.d=a(m, t, k, y)+d_{t, k}+1\right)$. Since $\operatorname{Res}_{Q}(X \cup J)=X$ and $h^{1}\left(\mathcal{I}_{X}(m-2)\right)=0$, we have $h^{1}\left(\mathcal{I}_{X \cup J}(m)\right)=0$. The union $X \cup J$ is a nodal and connected smoothable curve of degree $d$ and arithmetic genus $g$ and $Y \cup J$ is a 
smooth and connected curve of degree $d-d_{t, k}$ and arithmetic genus $g-g_{t, k}-2 \geq 26$. We may smooth $Y \cup J$ in a family of curves, all of them containing the two points $\left(C_{t} \cup C_{k}^{\prime}\right) \cap J$. Call $E$ a general element of this smoothing. Since $\operatorname{Aut}\left(\mathbb{P}^{3}\right)$ is 2transitive, we may see $E$ as a general non-special space curve of its degree and its genus $\geq 26$. By construction and Lemma 2 we have $C_{t} \cup C_{k}^{\prime} \cup E \in U\left(t, k, a_{d}, b\right)$ and $h^{1}\left(N_{C_{t} \cup C_{k}^{\prime} \cup E}(-1)\right)=0$. By semicontinuity there is a smooth $X_{1} \in W\left(t, k, a_{d}, b\right)$ with $h^{1}\left(\mathcal{I}_{X_{1}}(m)\right)=0$ and $h^{1}\left(N_{X_{1}}(-1)\right)=0$.

(a1.2) Assume $0<b(m-2, t, k, y) \leq z-3$. Hence $S_{2}=\emptyset$. We take $D_{1}$ and call $L_{i} \in\left|\mathcal{O}_{Q}(0,1)\right|, 1 \leq i \leq b(m-2, t, k, y)$, the elements of $\left|\mathcal{O}_{Q}(0,1)\right|$ such that $S_{1}=D_{1} \cap\left(L_{1} \cup \cdots \cup L_{b(m-2, t, k, y)}\right)$; note that each line $L_{i}$ contains a point of $Y \cap Q$. Take any $L_{b(m-2, t, k, y)+1} \in\left|\mathcal{O}_{Q}(0,1)\right|$ with $C_{k}^{\prime} \cap L_{b(m-2, t, k, y)+1} \neq \emptyset$, any $L_{b(m-2, t, k, y)+2} \in\left|\mathcal{O}_{Q}(0,1)\right|$ with $Y \cap L_{b(m-2, t, k, y)+2} \neq \emptyset, L_{b(m-2, t, k, y)+2} \neq L_{i}$ for $i \leq b(m-2, t, k, y)$ and (if $b(m-2, t, k, y)<z-3)$ take general $L_{j} \in\left|\mathcal{O}_{Q}(0,1)\right|$, $b(m-2, t, k, y)+3 \leq j \leq z-1$. Set $J:=D_{1} \cup\left(\bigcup_{i=1}^{z-1} L_{i}\right), \chi:=\cup_{o \in S_{1}} \chi(o)$ and $W:=X \cup J \cup \chi$. We have $\operatorname{Res}_{Q}(W)=X \cup S_{1}$ and thus $h^{1}\left(\mathcal{I}_{\operatorname{Res}_{Q}(W)}(m-2)\right)=0$. Since $\eta \geq 0$, (11) and the case $x=m-2$ of (11) give $2 d_{t, k}+2 a(m, t, k, y)-b(m-$ $2, t, k, y)-3=m(m+3-z)-\eta \leq h^{0}\left(Q, \mathcal{O}_{Q}(m-2, m+2-z)\right)$. Since $W \cap Q$ is the union of $J$ and $2 d_{t, k}+2 a(m, t, k, y)-b(m-2, t, k, y)-3$ general points of $Q$, we have $h^{1}\left(Q, \mathcal{I}_{W \cap Q}(m)\right)=h^{1}\left(Q, \mathcal{I}_{X \cap(Q \backslash J)}(m-1, m+1-z)\right)=0$. Thus $h^{1}\left(\mathcal{I}_{W}(m)\right)=0$. We first deform $W$ to the union $F$ of $C_{t} \cup C_{k}^{\prime} \cup D_{1} \cup Y \cup\left(\bigcup_{i=b(m-3, t, k, y)+1}^{z-1} L_{i}\right)$ and $b(m-3, t, k, y)$ disjoint lines $M_{1}, \ldots, M_{b(m-3, t, k, y)}$, each of them containing one point of $Y$. The union $F$ is a nodal and connected curve. Write $F=C_{t} \cup C_{k}^{\prime} \cup G$. We have $\sharp\left(G \cap C_{t}\right)=\sharp\left(G \cap C_{k}^{\prime}\right)=1$. Let $G^{\prime}$ be a general smoothing of $G$ fixing the 2 points of $\left(C_{t} \cup C_{k}^{\prime}\right) \cap G$. $C_{t} \cup C_{k}^{\prime} \cup G^{\prime} \in U\left(t, k, a_{d}, b\right)$. By Lemma 2 and semicontinuity there is a smooth $X_{2} \in W\left(t, k, a_{d}, b\right)$ with $h^{1}\left(\mathcal{I}_{X_{2}}(m)\right)=0$ and $h^{1}\left(N_{X_{2}}(-1)\right)=0$.

(a1.3) Assume $b(m-2, t, k, y) \geq z-2$. Since $z \geq a(m, t, k, y)-a(m-2, t, k)$ and $b(m-2, t, k, y) \leq m-3$, the case $x=m-2$ of Lemma 9 gives $2(z-3) \geq b(m-$ $2, t, k, y)$. Set $w:=b(m-2, t, k)-z+3$. Let $L_{i} \in\left|\mathcal{O}_{Q}(0,1)\right|, 1 \leq i \leq z-3$, be the line such that $S_{1}=D_{1}\left(\bigcup_{i=1}^{z-3} L_{i}\right)$ and $S_{2}:=D_{2} \cap\left(\bigcup_{i=1}^{w} L_{i}\right)$. Let $L_{z-2} \in\left|\mathcal{O}_{Q}(0,1)\right|$ be a line with $L_{z-2} \neq L_{i}$ for any $i \neq z-2$ and $L_{z-2} \cap Y \neq \emptyset$. Note that $L_{j} \cap Y \neq \emptyset$ if and only if either $j \leq w$ or $j=z-2$. Set $J:=D_{1} \cup D_{2} \cup\left(\bigcup_{i=1}^{z-2} L_{i}\right), \chi:=\cup_{o \in S_{1} \cup S_{2}} \chi(o)$ and $W:=X \cup J \cup \chi$ and continue as in the last step.

(a2) Assume $d<d(m, g)_{\max }$ We have $\eta \geq m\left(d(m, g)_{\max }-d\right) \geq m$ and in particular $\eta \geq m \geq b(m-2, t, k, y)+2$. To prove the existence of $X_{1}$ in this component we only need that $z \geq 3$, i.e. that $d \geq a_{m-2, t, k, y}+d_{t, k}+3$, which is true because $1+(m-1) d-g \geq\left(\begin{array}{c}m+2 \\ 3\end{array}\right)$ and $(m-1)\left(a(m-2, t, k, y)+d_{t, k}\right)+3-g=\left(\begin{array}{c}m+1 \\ 2\end{array}\right)-$ $a(m-2, t, k)-d_{t, k}+b(m-2, t, k, y) \geq 3 m$. Take $\left(X, Q, D_{1}, D_{2}, S_{1}, S_{2}\right)$ satisfying $N(m-2, t, k, y)$ with $X=C_{t} \sqcup C_{k}^{\prime} \sqcup Y$ and throw away $D_{1}, D_{2}, S_{1}$ and $S_{2}$. Fix $D \in\left|\mathcal{O}_{Q}(1,0)\right|$ containing one point of $Y \cap Q$ and $z-1$ distinct lines $L_{i} \in\left|\mathcal{O}_{Q}(0,1)\right|$ with $L_{i} \cap Y=\emptyset$ for all $i, L_{i} \cap C_{t} \neq \emptyset$ if and only if $i=1$ and $L_{i} \cap C_{k}^{\prime} \neq \emptyset$ if and only if $i=2$. Set $J:=D \cup\left(\bigcup_{i=1}^{z-1} L_{i}\right)$ and $W:=X \cup J$. As in the previous steps it is sufficient to prove that $h^{1}\left(\mathcal{I}_{W}(m)\right)=0$. We have $\operatorname{Res}_{Q}(W)=X$ and thus $h^{1}\left(\mathcal{I}_{\operatorname{Res}_{Q}(W)}(m-2)\right)=0$. Hence it is sufficient to prove that $h^{1}\left(Q, \mathcal{I}_{W \cap Q}(m)\right)=0$. We have $h^{1}\left(Q, \mathcal{I}_{Q \cap W}(m)\right)=h^{1}\left(Q, \mathcal{I}_{X \cap(Q \backslash J)}(m-1, m+1-z)\right)$. Since $X \cap Q$ is general in $Q$, it is sufficient to prove that $\sharp(X \cap(Q \backslash J)) \leq m(m+2-z)$. We have $\sharp(X \cap(Q \backslash J))=2 d_{t, k}+2 a(m-2, t, k, y)-3$. By the definition of $\eta$ and (10) for 
$x=m-2$ we have $2 d_{t, k}+2 a(m-2, t, k, y)-3=m(m+2-z)+b(m-2, t, k, y)+2-\eta \leq$ $m(m+2-z)$.

(b) In this part we get the existence of $A \in U\left(t, k, a_{d}, b\right)$ with $h^{0}\left(\mathcal{I}_{A}(m-\right.$ 1)) $=0, \operatorname{deg}(A)=d$ and $p_{a}(A)=g$, hence by semicontinuity the existence of $X_{2} \in W\left(t, k, a_{d}, b\right)$ with $h^{0}\left(\mathcal{I}_{X_{2}}(m-1)\right)=0$. We have $h^{i}\left(\mathcal{I}_{C_{t, k}}(t+k-1)\right)=0$, $i=0,1$ and $m-1 \equiv t+k(\bmod 2)$. Fix a plane $H$. Let $c$ be the maximal integer such that $\left(\begin{array}{c}t+k+2-c \\ 2\end{array}\right) \leq d_{t, k}$. Let $E \subset H$ be a general linear projection of a general smooth and rational degree $c$ curve $E^{\prime} \subset \mathbb{P}^{3}$. The curve $E$ is nodal and it has $(c-1)(c-2) / 2$ singular points. Set $\chi:=\cup_{p \in \operatorname{Sing}(E)} \chi(p)$. The union $E \cup \chi$ is the flat limit of a family of degree $c$ smooth rational curves in $\mathbb{P}^{3}$ (15, Fig. 11 at p. 260]. Hence to prove that a general union of some $C_{t, k}$ and a smooth rational curve of degree $c$ is contained in no surface of degree $t+k$ it is sufficient to prove that $h^{0}\left(\mathcal{I}_{C_{t, k} \cup E \cup \chi}(t+k)=0\right.$ for a general $C_{t, k}$. Thus it is sufficient to prove that $h^{0}\left(\mathcal{I}_{C_{t, k} \cup E}(t+k)\right)=0$ for a general $C_{t, k}$. For a general $C_{t, k}$ we have $C_{t, k} \cap E=\emptyset$ and $C_{t, k} \cap H$ is a general subset of $H$ with cardinality $d_{t, k}$. By definition $c$ is the minimal positive integer such that $h^{0}\left(H, \mathcal{I}_{C_{t, k} \cap H}(t+k-c)\right)=0$. Set $\beta=h^{0}\left(\mathcal{O}_{C_{t, k} \cup E \cup \chi}(t+k)\right)-\left(\begin{array}{c}t+k+3 \\ 3\end{array}\right)$. Since $\left(\begin{array}{c}t+k+2-c \\ 2\end{array}\right)-\left(\begin{array}{c}t+k-1 \\ 2\end{array}\right)=t+k+1-c$, we have $\beta \leq(c-1)(c-2) / 2+t+k+1-c$. Then we continue from the critical value $t+k$ to the critical value $t+k+2$ and so on.

At the end we obtain some $B \in U\left(t, k, a_{d}, b\right)$ with $h^{0}\left(\mathcal{I}_{B}(m-1)\right)=0$ if $1+$ $d(m-1)-g \geq\left(\begin{array}{c}m+2 \\ 3\end{array}\right)+\beta$. In particular it is sufficient to assume $d \geq d(m, g)_{\min }+$ $\lceil\beta /(m-1)\rceil$. We have $c \sim \sqrt{2} t$, because $\operatorname{deg}\left(C_{t, k}\right) \sim t^{2}$ and $\left(\begin{array}{c}t+k+2 \\ 2\end{array}\right) \sim 2 t^{2}$. Hence $\beta \sim(c-1)(c-2) / 2 \sim t^{2}$. Since $t \leq m / 4$, it is sufficient to have roughly $d \geq d(m, g)_{\min }+m / 4$.

Lemma 15. Fix $t$ and $k \in\{t-1, t\}$ such that $y \equiv t+k-1(\bmod 2)$ and let $g_{t, k}+g(t+k+5, t, k) \leq g \leq-1+g_{t+1, k+1}+g(t+k+7, t+1, k+1)$. Then we have $y \leq \sqrt{20} t-1$. In particular, if $t \geq\lfloor m / \sqrt{20}\rfloor-5$ then $y \leq m-6$.

Proof. We have $g_{t+1, k+1}-g_{t, k}=2 t^{2}-2$ if $k=t$ and $g_{t+1, k+1}-g_{t, k}=2 t^{2}-$ $2 t-1$ if $k=t-1$. For all integer $x \geq t+k+1$ such that $x \equiv t+k+1$ $(\bmod 2)$ we have $c(x, t, k)-c(x-2, t, k)) \geq(x+2) / 2($ Lemma 4). Remark 4 gives $c(t+k+1, t, k)=k+3$. By the definition of $y$, we have $y \geq k+t+5$ and $g \geq g_{t, k}+g(y, t, k)=g_{t, k}+c(y, t, k)-3(y-t-k-1) / 2-3 \geq g_{t, k}-3(y-t-$ $k-1) / 2+k+\sum_{i=1}^{(y-t-k-1) / 2}(c(t+k+1+2 i, t, k)-c(t+k+1+2 i-2, t, k)) \geq$ $g_{t, k}-3(y-t-k-1) / 2+k+(t+k+y+7)(y-t-k-1) / 8$. On the other hand, we have $g \leq-1+g_{t+1, k+1}+g(t+k+7, t+1, k+1) \leq-1+g_{t+1, k+1}+3(t+k+7)$. Hence we get $(t+k+y+7)(y-t-k-1) / 8 \leq g_{t+1, k+1}-g_{t, k}+3(y-t-k-1) / 2-k-1+3(t+k+7)$ and in particular $(y+1)^{2} \leq 20 t^{2}$.

Proof of Theorem [1: We fix the integer $g$ and we perform the above construction in both the odd and the even case, by taking either $k=t$ or $k=t-1$. We have $h^{1}\left(\mathcal{O}\left(C_{t, k}(t-1)=0\right.\right.$, hence we get $h^{1}\left(\mathcal{O}\left(C_{X}(t-1)=0\right.\right.$ by a repeated application of Mayer-Vietoris and semicontinuity. For every $t \geq 27$ such that $g \geq g_{t+3, k+3} \geq$ $g_{t, k}+g(t+k+5, t, k)$ we get an integer $y \equiv t+k-1$ such that the statement of Theorem 1 holds for every $m \geq y+6$ with $m \equiv y(\bmod 2)$. By Lemma 15, the condition $m \geq y+6$ is satisfied for every $t \geq\lfloor m / \sqrt{20}\rfloor-5$, hence we obtain our statement for every $g$ with $2 g_{30}=17052 \leq g \leq \varphi(m)$. 
Proof of Corollary [1: Let $m$ be the minimal non-negative integer such that

$$
m d+1-g \leq\left(\begin{array}{c}
m+3 \\
3
\end{array}\right)
$$

The minimality of $m$ gives

$$
(m-1) d+1-g>\left(\begin{array}{c}
m+2 \\
3
\end{array}\right)
$$

in particular $d \geq \frac{(m+2)(m+1) m}{6(m-1)} \geq \frac{m^{2}}{6}$. From (16) and (17) we get $d \leq\left(\begin{array}{c}m+2 \\ 2\end{array}\right)$. Since $g \leq K d^{3 / 2}-6 \varepsilon d$, we have

$$
\begin{aligned}
g & \leq \frac{2}{3}\left(\frac{1}{10}\right)^{3 / 2}\left(\begin{array}{c}
m+2 \\
2
\end{array}\right)^{3 / 2}-6 \varepsilon d \\
& \leq \frac{2}{3}\left(\frac{1}{10}\right)^{3 / 2}\left(\frac{(m+2)^{2}}{2}\right)^{3 / 2}-6 \varepsilon d \\
& \leq \frac{2}{3}\left(\frac{1}{20}\right)^{3 / 2}(m+2)^{3}-\varepsilon m^{2} \leq \varphi(m)
\end{aligned}
$$

(notice that the coefficients of $m^{3}$ are controlled by our choice of $K$ and the coefficients of $m^{2}$ are controlled by our choice of $\varepsilon$ ). Since $g \leq \varphi(m)$, Theorem 1 covers all degrees $d_{0}$ in the interval $d(m, g)_{\min } \leq d_{0} \leq d(m, g)_{\max }$. In order to check that $d$ is in this interval, just notice that $d \geq d(m, g)_{\min }$ by (17) and $d \leq d(m, g)_{\max }$ by (16).

\section{REFERENCES}

[1] E. Ballico and $\mathrm{Ph}$. Ellia, The maximal rank conjecture for non-special curves in $\mathbf{P}^{3}$. Invent. Math. 79 (1985), 541-555.

[2] E. Ballico and Ph. Ellia, Beyond the maximal rank conjecture for curves in $\mathbb{P}^{3}$, in: Space Curves, Proceedings Rocca di Papa, pp. 1-23, Lecture Notes in Math. 1266, Springer, Berlin, 1985.

[3] E. Ballico and Ph. Ellia, A program for space curves. Conference on algebraic varieties of small dimension (Turin, 1985). Rend. Sem. Mat. Univ. Politec. Torino 1986, Special Issue, 25-42 (1987).

[4] E. Ballico, G. Bolondi, Ph. Ellia and R. M. Mirò-Roig, Curves of maximum genus in the range A and stick-figures, Trans. Amer. Math. Soc. 349 (1997), 4589-4608.

[5] E. Carlini, M. V. Catalisano and A. Geramita, Bipolynomial Hilbert functions, J. Algebra 324 (2010), 758-781.

[6] K. Dasaratha, The reducibility and dimension of Hilbert schemes of complex projective curves, Undergraduate Thesis, Harvard University, Department of Mathematics, available at http://www.math.harvard.edu/theses/senior/dasaratha/dasaratha

[7] L. Ein, Hilbert scheme of smooth space curves. Ann. Sci. École Norm. Sup. (4) 19 (1986), no. 4, 469-478.

[8] L. Ein, The irreducibility of the Hilbert scheme of smooth space curves, Algebraic geometry, Bowdoin, 1985 (Brunswick, Maine, 1985), 83-87, Proc. Sympos. Pure Math., 46, Part 1, Amer. Math. Soc., Providence, RI, 1987.

[9] D. Eisenbud and A. Van de Ven, On the normal bundle of smooth rational space curves, Math. Ann. 256 (1981), no. 4, 453-463.

[10] Ph. Ellia, Exemples de courbes de $\mathbb{P}^{3}$ à fibré normal semi-stable, stable, Math. Ann. 264 (1983), 389-396.

[11] Ph. Ellia, Sur le genre maximal des courbes gauches de degré $d$ non sur une surface de degré $s-1$, J. Reine Angew. Math. 413 (1991), 77-87.

[12] G. Ellingsrud and A. Hirschowitz, Sur le fibré normal des courbes gauches, C. R. Acad. Sci. Paris 299 (1984), 245-248. 
[13] G. Fløystad, Construction of space curves with good properties, Math. Ann. 289 (1991), 33-54.

[14] G. Fløystad, On space curves with good cohomological properties, Math. Ann. 291 (1991), 505-549.

[15] R. Hartshorne, Algebraic Geometry, Springer-Verlag, Berlin-Heidelberg-New York, 1977.

[16] R. Hartshorne, On the classification of algebraic space curves, in: Vector bundles and differential equations (Nice 1979), p. 82-112, Progress in Math. 7, Birkhäuser, Boston 1980.

[17] R. Hartshorne and A. Hirschowitz, Droites en position générale dans l'espace projectif. Algebraic geometry (La Rábida, 1981), 169-188, Lecture Notes in Math., 961, Springer, Berlin, 1982 .

[18] R. Hartshorne and A. Hirschowitz, Smoothing algebraic space curves, Algebraic Geometry, Sitges 1983, 98-131, Lecture Notes in Math. 1124, Springer, Berlin, 1985.

[19] A. Hirschowitz, Sur la postulation générique des courbes rationnelles. Acta Math. 146 (1981), 209-230.

[20] A. Hirschowitz, Sections planes et multisécantes pour les courbes gauches génériques principales, Space curves (Rocca di Papa, 1985), 124-155, Lecture Notes in Math. 1266, Springer, Berlin, 1987.

[21] H. Iliev, On the irreducibility of the Hilbert scheme of space curves, Proc. Amer. Math. Soc. 134 (2006), 2823-2832.

[22] D. Jensen and S. Payne, Tropical independence II: The maximal rank conjecture for quadrics, Algebra Number Theory 10 (2016), no. 8, 1601-1640.

[23] C. Keem, Reducible Hilbert scheme of smooth curves with positive Brill-Noether number, Proc. Amer. Math. Soc. 122 (1994), 349-354.

[24] C. Keem and Y.-H. Kim, Irreducibility of the Hilbert scheme of smooth curves in $\mathbb{P}^{3}$ of degree $g$ and genus $g$, Arch. Math. 108 (2017), 593-600.

[25] C. Keem, Y.-H. Kim, and A.F. Lopez, Irreducibility and components rigid in moduli of the Hilbert Scheme of smooth curves, arXiv:1605.00297 (2016).

[26] C. Keem and S. Kim, Irreducibility of a subscheme of the Hilbert scheme of complex space curves, J. Algebra 145 (1992), 240-248.

[27] E. Larsen, The Maximal Rank Conjecture, arXiv: 1711:04906 (2017).

[28] T. Le Tat, Space curves with the expected postulation, J. Pure Appl. Algebra 77 (2003), $253-285$.

[29] D. Perrin, Courbes passant par $m$ points généraux de $\mathbb{P}^{3}$, Bull. Soc. Math. France, Mémoire 28/29 (1987).

[30] E. Sernesi, On the existence of certain families of curves, Invent. Math. 75 (1984), 25-57.

[31] Ch. H. Walter, Curves in $\mathbb{P}^{3}$ with the expected monad, J. Algebraic Geom. 4 (1995), 301-320.

(Edoardo Ballico, Claudio Fontanari) Dipartimento di Matematica, Università di Trento, Via Sommarive 14, 38123 Povo (Trento), Italy.

E-mail address: edoardo.ballico@unitn.it, claudio.fontanari@unitn.it

(Philippe Ellia) Dipartimento di Matematica e Informatica, Università degli Studi di Ferrara, Via Machiavelli 30, 44121 Ferrara, Italy.

E-mail address: phe@unife.it 